Article

\title{
Squint Mode GEO SAR Imaging Using Bulk Range Walk Correction on Received Signals
}

\author{
Jiwen Geng ${ }^{1}$, Ze Yu ${ }^{1}{ }^{1 *}$, Chunsheng $\mathrm{Li}^{1}$ and Wei Liu ${ }^{2}$ (D) \\ 1 School of Electronic and Information Engineering, Beihang University, Beijing 100191, China; \\ gengjiwen@buaa.edu.cn (J.G.); chunshengli201@163.com (C.L.) \\ 2 Department of Electronic \& Electrical Engineering, University of Sheffield, Sheffield S1 4ET, UK; \\ w.liu@sheffield.ac.uk \\ * Correspondence: yz613@buaa.edu.cn; Tel.: +86-185-1920-7286
}

Received: 11 September 2018; Accepted: 19 December 2018; Published: 21 December 2018

\begin{abstract}
Geosynchronous synthetic aperture radar (GEO SAR) has the potential for conducting long-term observation of target zones, which is essential for remote sensing applications such as disaster monitoring and vegetation measurements. The squint imaging mode is crucial for long-term observation using GEO SAR. However, this type of SAR imaging is problematic because the squint mode introduces a nonzero range cell walk, which increases the prevalence of invalid data in echoes and intensifies the coupling between the azimuth and range. Therefore, this paper proposes a novel squint mode GEO SAR imaging method based on the correction of the bulk range walk of received signals. Adjusting the starting time of the receiving window significantly reduces the redundancy in echoes. Then, first-order filtering, range cell migration correction, range compression, partial dechirp, and azimuth compression are used to obtain the imaging result. Simulation results for the GEO SAR imaging of Wenchuan County in China demonstrate that the proposed algorithm can achieve a resolution of $5 \mathrm{~m}$ within a $30 \times 30 \mathrm{~km}$ swath over $48 \%$ of the orbital period.
\end{abstract}

Keywords: geosynchronous synthetic aperture radar; squint mode imaging; range walk correction

\section{Introduction}

Geosynchronous synthetic aperture radar (GEO SAR) operates on an inclined orbit at a speed of nearly $3.075 \mathrm{~km} / \mathrm{s}$ and at a height of approximately 35,786 km [1,2]. Compared with low earth orbit (LEO) SAR, GEO SAR moves more slowly and has a wider field of view that is nearly one-third of the earth's surface [3]. These features enable GEO SAR to observe target zones for a much longer period than LEO SAR, which is beneficial for conducting disaster monitoring [4]. Figure 1 illustrates an observation mode suitable for long-term observation via GEO SAR. This mode is composed of several strip-map observations acquired with different squint angles, and the target zone is imaged according to the overlapping area of the strip-map data received. Here, not only broadside SAR imaging, but also squint mode SAR imaging, must be used to process echoes acquired by this observation mode. The present study focuses on squint mode strip-map GEO SAR imaging. 


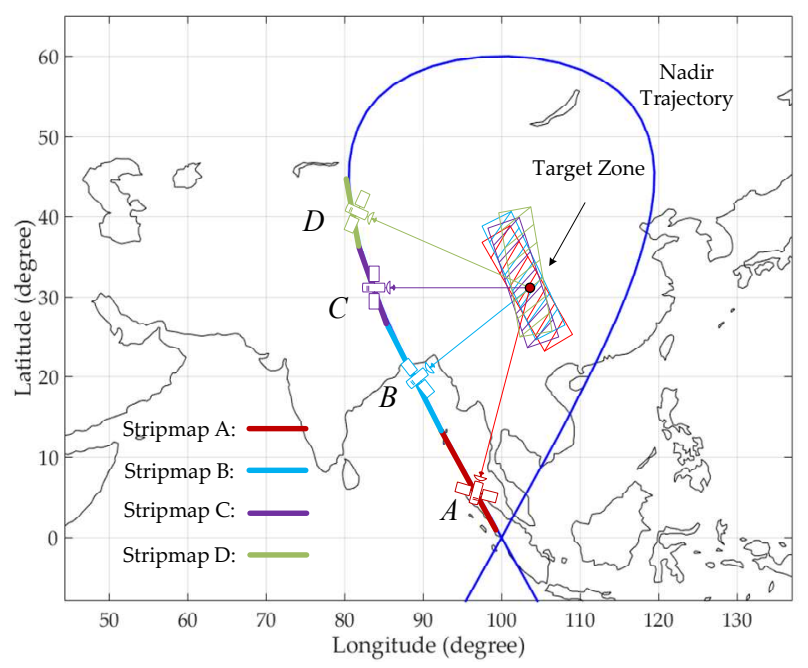

Figure 1. Illustration of a geosynchronous synthetic aperture radar (GEO SAR) observation mode. The red point is the center of the target zone. The blue line on the map represents the nadir trajectory of the GEO SAR platform. The trajectory includes four segments A, B, C, and D denoted by different colors that correspond to separate strip-map observations. A constant squint angle is adopted in each segment, which varies from segment to segment to ensure that the beam center coincides exactly with the red point when the platform resides at the center of each segment. Because the orbit of the GEO SAR platform is extremely high, the overlapping area of the four strip-map observations is sufficiently large to fully image the target zone.

The relative trajectories between GEO SAR platforms and targets are curved, which varies the range histories spatially in both the azimuth and range directions [5]. A number of GEO SAR imaging algorithms have been developed to correct this two-dimensional (2D) spatial variance. For example, the time-domain back projection (BP) algorithm has been developed for image focusing [6]. However, this algorithm suffers from its tremendous computational burden, even though the fast $\mathrm{BP}$ algorithm reduces the computational burden to $O\left(N^{5 / 2}\right)$ [7]. In contrast, the computational complexity of frequency-domain algorithms is much lower, and numerous algorithms that operate in this domain have been developed. The classic frequency-domain SAR imaging algorithms include the omega-K methods presented by Claudio Prati and Fabio Rocca [8], the methods based on the chirp scaling [9], and the frequency domain algorithms proposed by Franceschetti et al. [10]. As aforementioned, the severe spatial variance makes these classical algorithms fail in GEO SAR. However, inspired by these methods, some scholars developed algorithms for GEO SAR. Bao et al. [11] modified the chirp scaling (CS) algorithm based on a fourth-order polynomial model, and achieved an 8-m resolution within a $40 \times 40 \mathrm{~km}$ swath. Hu et al. [12] improved the conventional nonlinear CS algorithm by accounting for a linear spatial variance in the range direction in the polynomial model. Tao et al. [13] developed the nonlinear azimuth CS algorithm by introducing spatially variant components in the classic hyperbolic model. Sun et al. [14] considered spatial variation in both the range and azimuth directions in a fifth-order polynomial model, and a 5-m resolution within an $82 \times 95 \mathrm{~km}$ swath was achieved using range cell migration (RCM) equalization and sub-band synthesis technologies. To avoid the requirement for conducting sub-band synthesis, Chen et al. [15] combined singular value decomposition and azimuth nonlinear scaling, and obtained a 20-m resolution within a $150 \times 130 \mathrm{~km}$ swath. Yu et al. [16] proposed a time-frequency scaling algorithm to correct linear and quadratic variances in the azimuth direction and obtained well-focused results. Ding et al. [17] modified the quadratic term in the azimuth direction and obtained a 20-m resolution within a $400 \times 200 \mathrm{~km}$ swath under conditions where the Doppler rate was zero or approximately zero.

Notably, all of the aforementioned frequency-domain algorithms were designed for broadside GEO SAR imaging where the range cell walk is zero. However, the squint mode introduces a nonzero range walk, which increases the prevalence of invalid data in echoes, and intensifies the coupling 
between the azimuth and the range. As a result, squint mode SAR imaging is considerably more complicated than broadside imaging. Therefore, correcting the range walk is the key to developing high-resolution squint mode GEO SAR imaging. Whereas past efforts have addressed this issue using range migration removal and 2D nonlinear CS [18], the researchers assumed that the range walk was spatially invariant over the entire swath, which restricted the performance of the developed algorithm. Therefore, we propose a novel squint mode GEO SAR imaging method based on the correction of the bulk range walk of echoes. The key component of the method is to exploit the characteristics of the low pulse repetition frequency (PRF) employed in GEO SAR, and accordingly adjust the starting time of the receiving window. Then, first-order filtering, RCM correction (RCMC), range compression, partial dechirp, and azimuth compression are used to obtain the imaging result. Although the bulk range walk can be corrected in the signal processing stage, the proposed method conducts the correction while receiving the echoes (i.e., during receiving), which significantly reduces the echoes redundancy and improve the efficiency of on-board storage.

The remainder of this paper is structured as follows. Section 2 analyzes the range walk of squint mode GEO SAR based on a slant range model, and illustrates the advantages of conducting range walk removal upon receiving. Section 3 discusses the feasibility of conducting range walk removal on receiving by comparing the Doppler bandwidths of GEO SAR and LEO SAR, and presents the proposed approach for adjusting the starting time of the receive window. An imaging algorithm based on echoes with the bulk range walk corrected is then proposed in Section 4. Simulation results for the squint mode GEO SAR imaging of Wenchuan County in China are presented and analyzed in Section 5, and the results are discussed in Section 6. Finally, Section 7 concludes the paper.

\section{Slant Range History of Squint Mode GEO SAR}

Figure 2 illustrates the observation geometry between the strip-map GEO SAR imaging and a target zone as the GEO SAR platform moves along its orbit. At azimuth time $t_{\text {ref }}$, the beam center points to the reference target $P_{r e f}$, which is usually the center of the target zone, and the slant range between the beam center and $P_{\text {ref }}$ is denoted by $R_{r e f}$. At the azimuth time $t_{c}$, the beam center points to $P_{c}$, and the corresponding slant distance is $R_{c}$. The slant range history of GEO SAR imaging can be analyzed according to Figure 2 as follows.

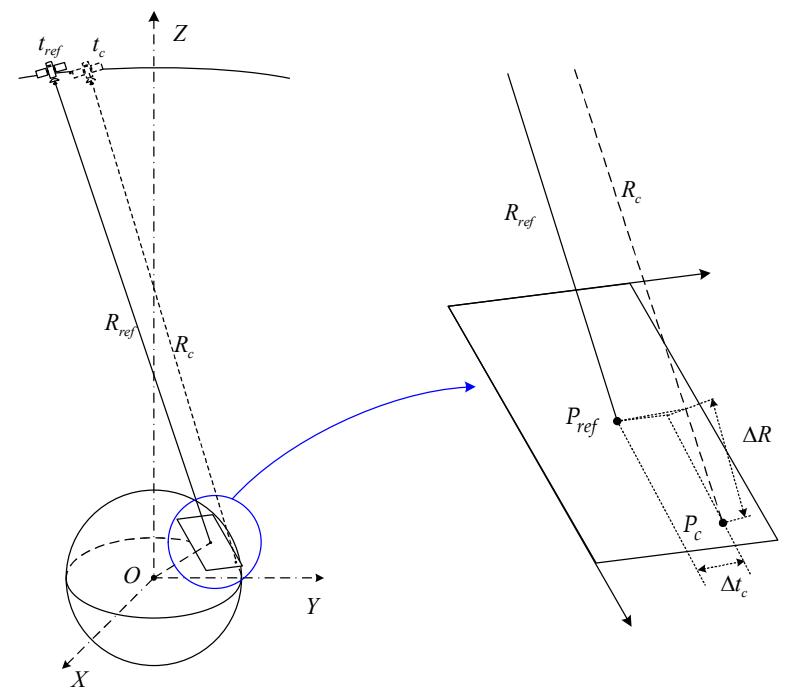

Figure 2. Observation geometry between strip-map GEO SAR imaging and a target zone as the GEO SAR platform moves along its orbit. The target zone area enclosed by the blue circle in the diagram on the left side is enlarged in the diagram on the right side. At $t_{r e f}$ and $t_{c}$, the beam center points to targets $P_{r e f}$ and $P_{c}$, respectively, where $P_{\text {ref }}$ is the reference target, which is also the center of the target zone. At $t_{r e f}$, the slant range between the beam center and $P_{r e f}$ is $R_{r e f}$. At $t_{c}$, the slant range between the beam center and $P_{c}$ is $R_{c}$. 
Ignoring the antenna weighting, the GEO SAR echo from $P_{c}$ can be expressed as:

$$
S_{\text {echo }}(t, \tau)=\sigma \cdot \operatorname{rect}\left(\frac{t-t_{c}}{T_{s}}\right) \cdot \operatorname{rect}\left[\frac{\tau-2 R(t) / c}{T_{p}}\right] \cdot \exp \left[j \pi K_{r}\left(\tau-\frac{2 R(t)}{c}\right)^{2}\right] \cdot \exp \left[-j \frac{4 \pi}{\lambda} R(t)\right],
$$

where $t$ and $\tau$ denote the slow time in the azimuth direction and the fast time in the range direction, respectively; $\sigma$ is the radar cross-section of $P_{c} ; \operatorname{rect}(\cdot)$ is the rectangle function; $T_{S}$ is the synthetic aperture time; $R(t)$ represents the slant range history between the beam center and $P_{c} ; c$ is the speed of light; $T_{p}$ is the pulse width of the transmitted chirp signal; $K_{r}$ is the frequency modulation rate; and $\lambda$ is the wavelength of the beam. As previously reported [16], $R(t)$ can be described by a polynomial expression of order $N$, as follows:

$$
R(t) \approx \sum_{n=0}^{N} \frac{r_{n}}{n !}\left(t-t_{c}\right)^{n}
$$

where $r_{n}$ is the $n$th polynomial coefficient that can be obtained by fitting based on ephemeris data and geographic information specific to the target zone. A value of $N=5$ has been demonstrated to be sufficiently large for GEO SAR imaging [16], and that value is assumed hereafter. However, because $R(t)$ varies for different target zones at different locations, the coefficients $r_{n}$ are spatially variant, and can be expressed as functions of $\Delta R=R_{c}-R_{r e f}$ and $\Delta t=t_{c}-t_{r e f}$, as follows:

$$
\left\{\begin{array}{l}
r_{0}=r_{0, \text { ref }}+\Delta R \\
r_{1}=r_{1, \text { ref }}+k_{1,1, r} \cdot \Delta R+\left.k_{1,1, a}\right|_{\Delta R} \cdot \Delta t \\
r_{2}=r_{2, \text { ref }}+k_{2,1, r} \cdot \Delta R+k_{2,2, r} \cdot \Delta R^{2}+\left.k_{2,1, a}\right|_{\Delta R} \cdot \Delta t+\left.k_{2,2, a}\right|_{\Delta R} \cdot \Delta t^{2} \\
r_{3}=r_{3, \text { ref }}+k_{3,1, r} \cdot \Delta R+\left.k_{3,1, a}\right|_{\Delta R} \cdot \Delta t+\left.k_{3,2, a}\right|_{\Delta R} \cdot \Delta t^{2} \\
r_{4}=r_{4, \text { ref }}+k_{4,1, r} \cdot \Delta R+\left.k_{4,1, a}\right|_{\Delta R} \cdot \Delta t \\
r_{5}=r_{5, \text { ref }}
\end{array}\right.
$$

where the auxiliary coefficients $r_{n, r e f}, k_{n, m, r}$, and $\left.k_{n, m, a}\right|_{\Delta R}$, where $\left.k_{n, m, a}\right|_{\Delta R}$ denotes the spatially variant coefficient $k_{n, m, r}$ at $\Delta R$, can be calculated as presented previously in Yu et al. [16].

As Figure 3 shows, echoes usually contain range walk $\Delta R_{W}$ and range curvature $\Delta R_{B}$. According to Equation (2), the first-order coefficient $r_{1}$ mainly causes the range walk. Therefore, the range walk and the range curvature are given as follows:

$$
\left\{\begin{array}{l}
\Delta R_{W}=r_{1} T_{s} \approx r_{1, r e f} T_{s} \\
\Delta R_{B}=\max _{t \in T_{s}}\left\{R(t)-\Delta R_{W}-R_{c}\right\} \approx r_{2, r e f} T_{s}^{2} / 8
\end{array}\right.
$$

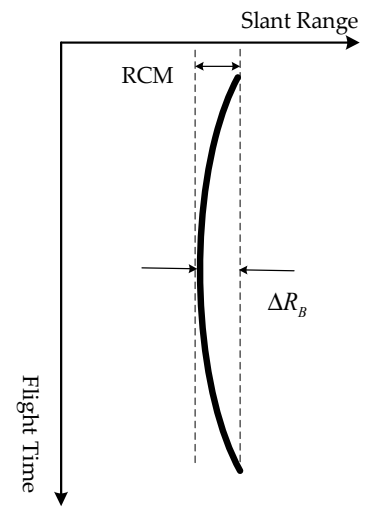

(a)

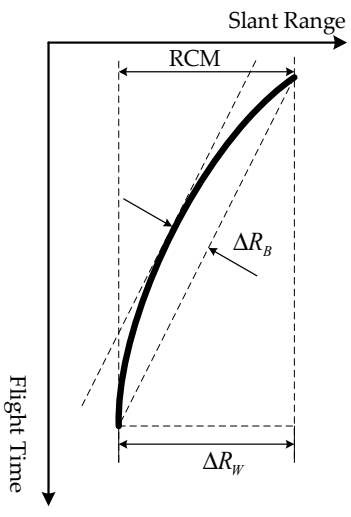

(b)

Figure 3. Range cell migration (RCM) of GEO SAR: (a) Broadside looking mode; (b) squint looking mode. 
In frequency domain squint mode GEO SAR imaging, both $\Delta R_{W}$ and $\Delta R_{B}$ should be corrected to make the migration curve limited in one single range bin.

To evaluate the proportion of $\Delta R_{W}$ requiring correction, we define a ratio $\chi_{W}$ as:

$$
\chi_{W}=\frac{\Delta R_{W}}{\Delta R_{W}+\Delta R_{B}} \approx \frac{8 r_{1, r e f}}{8 r_{1, r e f}+r_{2, r e f} T_{S}} .
$$

Figure 4 illustrates the value of $\chi_{W}$ obtained for different squint angles $\theta_{s}$, where the GEO SAR platform operates on an orbit with an inclination angle of $60^{\circ}$. Here, we note that $\chi_{W}$ generally increases with increasing $\theta_{s} . \chi_{W}$ is greater than $90 \%$ for the most of the orbital period. This indicates that $\Delta R_{W}$ accounts for the vast majority of the correction required. We note that for a swath of width $\Delta W$, the volume of recorded echo data can be given as:

$$
V_{\text {echo }} \approx \frac{2}{c}\left(\Delta W \cdot \sin \theta_{\text {inc }}+\frac{c T_{p}}{2}+\max \left\{\Delta R_{B}, \Delta R_{W}\right\}\right) \cdot F_{s} \cdot N_{a},
$$

where $\theta_{\text {inc }}$ is the incidence angle corresponding to the center of the target zone, $F_{S}$ is the range sampling rate, and $N_{a}$ is the azimuth sampling rate. From Equation (5), the ratio $\Delta R_{W} / \Delta R_{B}$ is given by $\chi_{W} /\left(1-\chi_{W}\right)$, which means $\Delta R_{W}$ is much higher than 9 times of $\Delta R_{B}$. Equation (6) indicates that $V_{\text {echo }}$ is significantly reduced if $\Delta R_{W}$ is corrected on receiving rather than storing the data for later image processing. Consequently, the efficiency of on-board GEO SAR data storage increases.

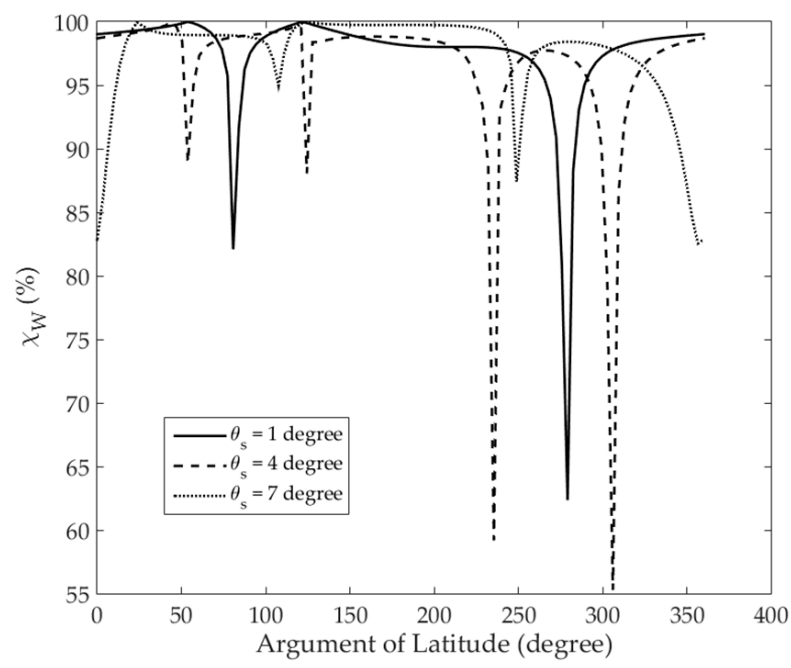

Figure 4. Values of ratio $\chi_{W}$, defined in Equation (5), obtained with different squint angles $\theta_{s}$, which include 1,4 , and 7 degrees. Because of the high orbital height, the maximum value of $\theta_{S}$ is approximately $7^{\circ}$ for GEO SAR.

\section{Bulk Range Walk Correction on Receive}

Compared with LEO SAR, the Doppler band of GEO SAR is much narrower. This feature makes it feasible to conduct bulk range walk correction by adjusting the starting time of the receiving window. The specific features of GEO SAR and the proposed adjustment principle are presented in the following discussion.

\subsection{Doppler Bandwidth of GEO SAR}

Since the relative trajectory between a GEO SAR platform and a target zone is curved, the azimuth resolution is no longer inversely proportional to the Doppler bandwidth. The relationship between the Doppler bandwidth and azimuth resolution is established in the wavenumber domain in this paper. 
As illustrated in Figure 5, a GEO SAR platform moves from $S_{1}$ to $S_{2}$ while observing $P_{c}$. $S_{0}$ is the midpoint of the arc $S_{1} S_{2}$. The corresponding Doppler bandwidth is:

$$
B_{a}=\left|f_{d 2}-f_{d 1}\right|,
$$

where $f_{d 1}$ and $f_{d 2}$ are the instantaneous Doppler frequencies at $S_{1}$ and $S_{2}$, respectively, which are given as follows:

$$
\begin{aligned}
& f_{d 1}=(2 / \lambda) \cdot \vec{V}_{s t 1} \cdot\left(\vec{R}_{t}-\vec{R}_{s 1}\right) /\left|\vec{R}_{t}-\vec{R}_{s 1}\right| \\
& f_{d 2}=(2 / \lambda) \cdot \vec{V}_{s t 2} \cdot\left(\vec{R}_{t}-\vec{R}_{s 2}\right) /\left|\vec{R}_{t}-\vec{R}_{s 2}\right|
\end{aligned}
$$

where $\overrightarrow{V_{s t 1}}$ and $\overrightarrow{V_{s t 2}}$ are the respective relative velocity vectors between the GEO SAR platform and $P_{c}$ at $S_{1}$ and $S_{2}, \overrightarrow{R_{s 1}}$ and $\overrightarrow{R_{s 2}}$ are the respective platform position vectors at $S_{1}$ and $S_{2}$, and $\overrightarrow{R_{t}}$ is the position vector of $P_{c}$. In Figure $5, \overrightarrow{i_{s t 0}}, \overrightarrow{i_{s t 1}}$, and $\overrightarrow{i_{s t 2}}$ are the unit vectors from $P_{c}$. to $S_{0}, S_{1}$, and $S_{2}$, respectively. The wavenumber vectors corresponding to $\overrightarrow{i_{s t 0}}, \overrightarrow{i_{s t 1}}$, and $\overrightarrow{i_{s t 2}}$ are given as follows, respectively:

$$
\overrightarrow{k_{r 0}}=\frac{4 \pi}{\lambda} \overrightarrow{i_{s t 0}}, \overrightarrow{k_{r 1}}=\frac{4 \pi}{\lambda} \overrightarrow{i_{s t 1}} \text {, and } \overrightarrow{k_{r 2}}=\frac{4 \pi}{\lambda} \overrightarrow{i_{s t 2}} \text {. }
$$

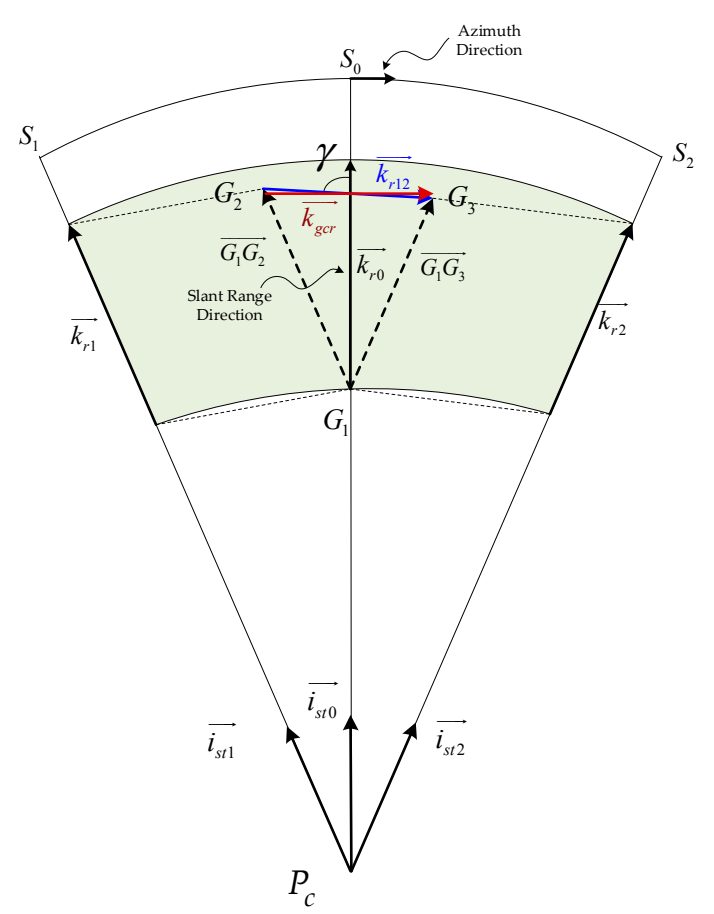

Figure 5. GEO SAR observation of target $P_{c}$ in the wavenumber domain. Here, $S_{0}, S_{1}$, and $S_{2}$, are different positions of the GEO SAR satellite. $\overrightarrow{i_{s t 0}}, \overrightarrow{i_{s t 1}}$, and $\overrightarrow{i_{s t 2}}$ are unit vectors from $P_{c}$ to $S_{0}, S_{1}$, and $S_{2}$, respectively, and $\overrightarrow{k_{r 0}}, \overrightarrow{k_{r 1}}$, and $\overrightarrow{k_{r 2}}$ are the wavenumber vectors in the corresponding directions. The vector $\overrightarrow{G_{1} G_{2}}$ is parallel to $\overrightarrow{k_{r 1}}$, and $\left|\overrightarrow{G_{1} G_{2}}\right|=\left|\overrightarrow{k_{r 1}}\right|$. The vector $\overrightarrow{G_{1} G_{3}}$ is parallel to $\overrightarrow{k_{r 2}}$, and $\left|\overrightarrow{G_{1}} \overrightarrow{G_{3}}\right|=\left|\overrightarrow{k_{r 2}}\right|$. The blue and red arrows represent $\overrightarrow{k_{r 12}}=\overrightarrow{k_{r 2}}-\overrightarrow{k_{r 1}}$ and $\overrightarrow{k_{g c r}}$, respectively, where $\overrightarrow{k_{g c r}}$ is the projection of $\overrightarrow{k_{r 12}}$ in the azimuth direction. 
In the wavenumber domain, the azimuth resolution $\rho_{a}$ is determined by the azimuth wavenumber vector $\overrightarrow{k_{g c r}}$, which is represented by the red arrow in Figure 5 . Here, $\overrightarrow{k_{g c r}}$ is the projection of the vector $\overrightarrow{k_{r 12}}=\overrightarrow{k_{r 2}}-\overrightarrow{k_{r 1}}$ in the azimuth direction. Therefore, $\overrightarrow{k_{g c r}}$ can be given as:

$$
\overrightarrow{k_{g c r}}=\overrightarrow{k_{r 12}} \cdot \sin \gamma
$$

where $\gamma$ is the angle between $\overrightarrow{k_{r 0}}$ and $\overrightarrow{k_{r 12}}$ and is defined as:

$$
\gamma=\arcsin \left\{\sqrt{1-\left[\overrightarrow{i_{s t 0}} \cdot\left(\overrightarrow{i_{s t 2}}-\overrightarrow{i_{s t 1}}\right)\right]^{2}}\right\} .
$$

Accordingly, the azimuth resolution can be calculated as:

$$
\rho_{a}=\frac{2 \pi}{k_{g c r}}=\frac{\lambda}{2\left|\overrightarrow{i_{s t 2}}-\overrightarrow{i_{s t 1}}\right| \cdot \sin \gamma} .
$$

The relationship between azimuth resolution and Doppler bandwidths varies with different targets at different orbit positions. Equations (7) and (10) provide a solution. Using the iterative method to determine the starting observation position (e.g., $S_{1}$ in Figure 5) and ending observing position (e.g., $S_{2}$ in Figure 5) under the predetermined azimuth resolution, the Doppler bandwidths can be obtained by Equation (7). Figure 6 presents the Doppler bandwidths of GEO SAR and LEO SAR with an azimuth resolution of $5 \mathrm{~m}$. The figure indicates that the Doppler bandwidth of GEO SAR is much smaller than that of LEO SAR, which is caused by the slower relative speed between GEO SAR platforms and targets.

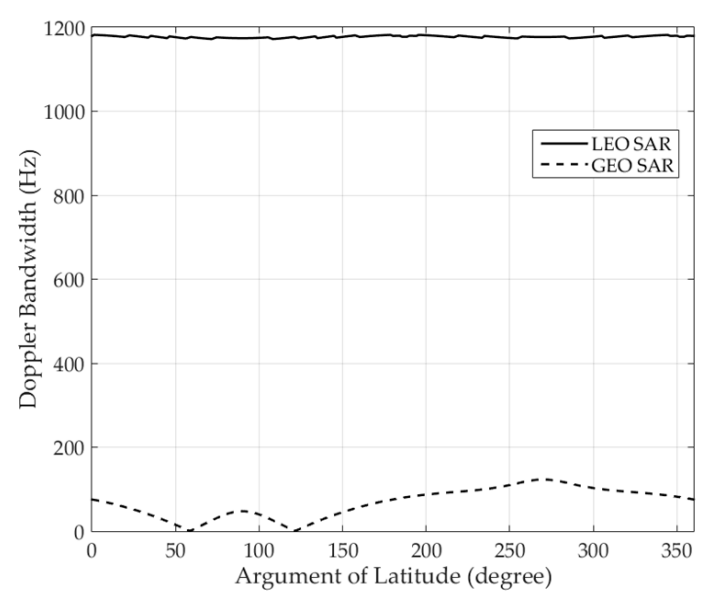

Figure 6. Doppler bandwidths of GEO SAR and low earth orbit (LEO) SAR with an azimuth resolution of $5 \mathrm{~m}$ based on Equations (7) and (10). We consider L-band GEO SAR in orbit with an inclination angle of $60^{\circ}$. The parameters employed for LEO SAR are the same as those of L-band ALOS-2 [19]. In this case, the Doppler bandwidth of GEO SAR is nearly 1/10 that of LEO SAR.

\subsection{Adjusting the Starting Time of the Receive Window}

Spaceborne SAR transmits signals at the PRF, which is usually 1.1-1.2 times the Doppler bandwidth [20]. The echo is recorded between two adjacent transmission pulses in the receive window. The starting time of the receive window determines the nearest range at which the beginning of the echo can be recorded. Therefore, adopting a variable starting time can alter the form of the recorded data. This is illustrated in Figure 7 for the echo data obtained from a single isolated point target when employing a constant starting time given by the blue line in Figure $7 \mathrm{a}$, and when employing 
the variable starting time, given by the red line in Figure $7 \mathrm{~b}$. We note that adjusting the starting time greatly reduces the RCM corresponding to the target, which indicates that the echoes can be recorded using less on-board storage and the range-azimuth coupling decreased. To avoid overlapping between the transmitted pulses and the received signals, the scope for adjusting the starting time must satisfy

$$
\Delta t_{a}<P R T-T_{p}
$$

where the pulse repetitive time is $P R T=1 / P R F$. For LEO SAR with a resolution of $5 \mathrm{~m}, \Delta t_{a}$ is less than $0.7 \mathrm{~ms}$ [21], which provides no scope for adjusting the starting time. However, the much lower PRF employed with GEO SAR relative to that employed with LEO SAR provides a value of $\Delta t_{a}$ as long as $12 \mathrm{~ms}$, which provides ample scope for adjusting the starting time of the receive window.

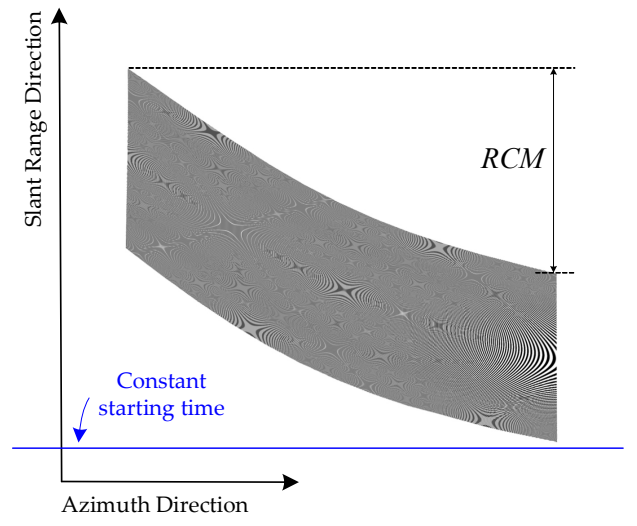

(a)

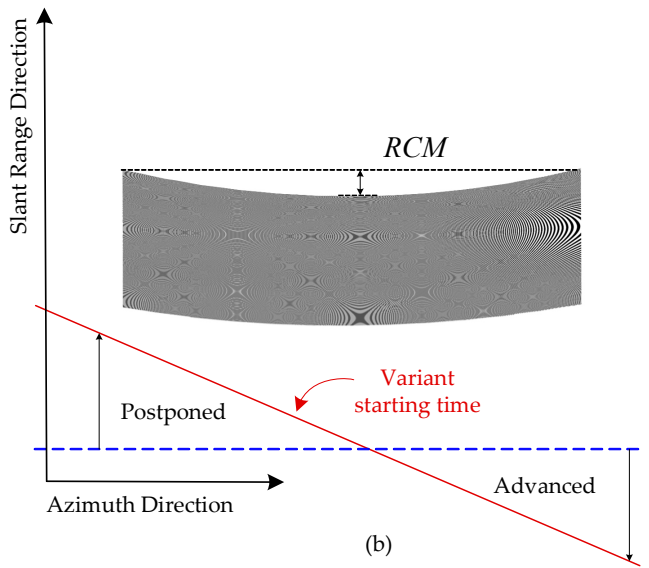

(b)

Figure 7. Effect of a variable starting time for the receive window. (a) The echo acquired by the squint mode with a constant starting time for the receive window represented by the blue line. (b) The echo acquired using the same mode but with a starting time that varies according to the red line, where the starting time is postponed during the first half of data recording and is advanced during the latter half.

In this paper, we adjusted the starting time to correct the bulk range walk of the reference target, which is more than $90 \%$ of the sum of $\Delta R_{W}$ and $\Delta R_{B}$ for an entire swath. Since the range walk is directly defined by $r_{1}$ in Equation (4), the range walk corrected means this item is removed. Therefore, the slant range of a reference target after correction becomes:

$$
R_{\text {ref_RWC }}(t)=r_{0 \_r e f}+\sum_{n=2}^{5} \frac{r_{n}^{n}}{n !} t^{n} .
$$

By comparing Equations (2) and (12), we note that the linear component representing the range migration disappears. Therefore, the adjusted starting time $T_{S_{-} R W C}$ should satisfy:

$$
T_{S_{-} R W C}=T_{S_{-} \text {general }}+2\left[R_{\text {ref_RWC }}(t)-R_{\text {ref_act }}(t)\right] / c,
$$

where $T_{S_{-} \text {general }}$ is the starting time before adjustment and $R_{\text {ref_RWC }}(t)$ is the actual range history of the reference target. Furthermore, correcting the bulk range walk on receive ensures that $r_{1, \text { ref }}$ in Equation (3) is equal to 0, which indicates that:

$$
r_{1}=k_{1,1, r} \cdot \Delta R+\left.k_{1,1, a}\right|_{\Delta R} \cdot \Delta t
$$

Equation (13) can be further explained based on the observation geometry illustrated in Figure 8. Here, GEO SAR observes the reference target $P_{\text {ref }}$ with a squint angle $\theta_{s}$. The echoes are sampled in fast and slow time. Here, fast time occurs in the beam pointing direction, which is also the direction of transmission and reception, and slow time occurs in the flight direction, i.e., the azimuth direction, 
prior to adjusting the starting time of the receive window. By applying Equation (13), the receive time is postponed from $A$ to $A^{\prime}$ or advanced from $B$ to $B^{\prime}$. As a result, slow time is changed from the flight direction to the new direction denoted by the red dotted line. As such, the deviation between the red dotted line and the flight trajectory complies with Equation (13). The operation makes the new slow time and fast time directions orthogonal, and changes the squint mode to an equivalent broadside mode. However, this change from squint mode to broadside mode is only applicable to $P_{\text {ref, }}$ and the other targets in the swath are still subject to a slight degree of squint mode observation.

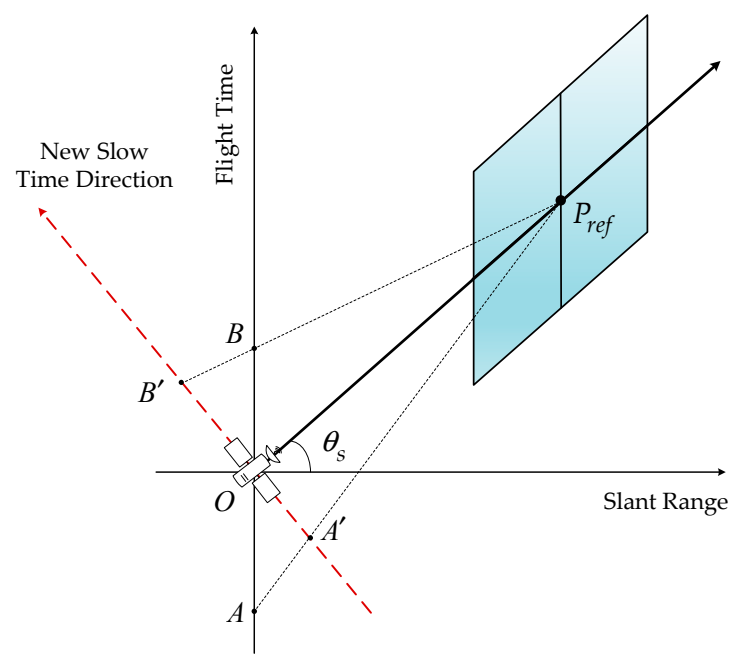

Figure 8. Equivalence between the broadside mode and the squint mode for a reference target $P_{\text {ref }}$ using a variable starting time for the receive window.

\section{Squint Imaging Algorithm for GEO SAR after Correcting Bulk Range Walk}

The proposed imaging process comprising operations in the range and azimuth directions after correcting the bulk range walk on receive is illustrated in Figure 9. The echo data of four isolated point targets with bulk range walk removed is shown in Figure 9a. First-order filtering was applied in the range direction to compensate for the range-dependent term $k_{1,1, r} \cdot \Delta R$ in Equation (14), as shown in Figure $9 \mathrm{~b}$. Then, RCMC and range compression were implemented to obtain the signal illustrated in Figure 9c. Next, partial dechirp was used to shorten the span of the aperture time for every target to facilitate the azimuthal segmentation of echoes. The results are shown in Figure 9d. Then, azimuth compensation was conducted, which led to the imaging result illustrated in Figure 9e. The proposed squint mode imaging algorithm is described by the flowchart given in Figure 10. The detailed steps in the algorithm are outlined in the following subsections.

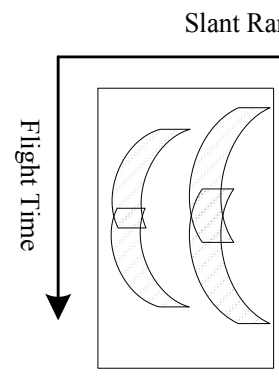

(a)

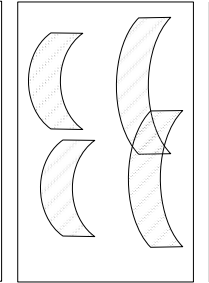

(b)

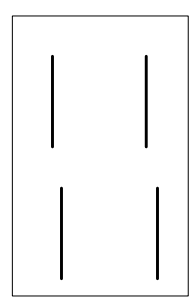

(c)

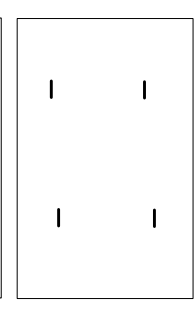

(d)

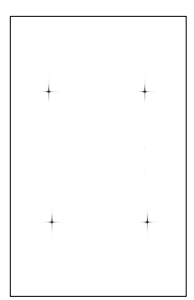

(e)

Figure 9. Conceptual basis for the proposed squint mode GEO SAR imaging algorithm. The echo signals after bulk range walk correction are demonstrated as subfigure (a). Then first-order filtering is applied, and the results are presented as (b). RCMC and other range operations together make the signals into (c). Partial dechirp and relevant operations shorten the azimuth span, which is shown in (d). Lastly, azimuth compensation produces the final images (e). 


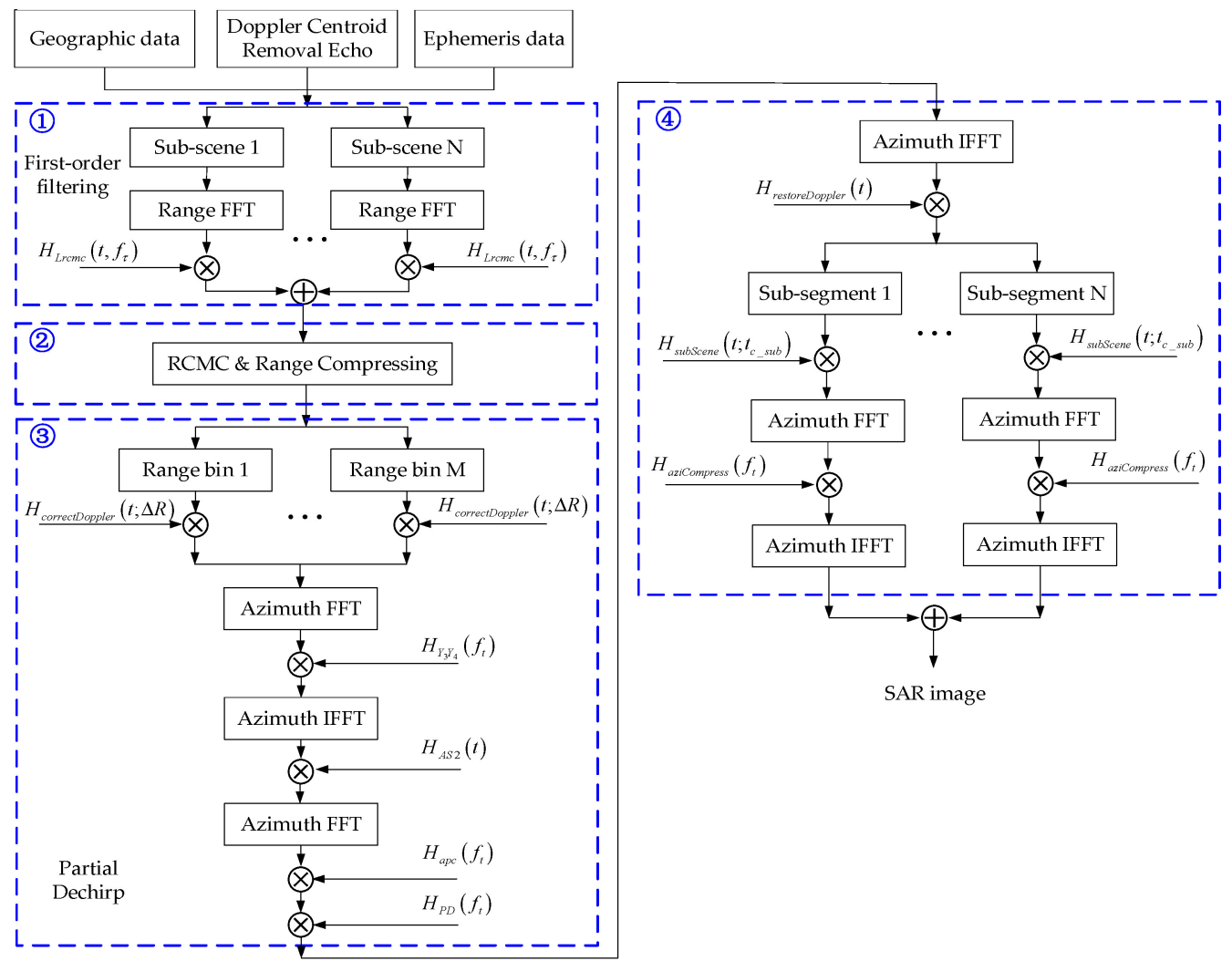

Figure 10. Flowchart for the proposed squint mode GEO SAR imaging algorithm, which is composed of four steps. Step 1 is first-order filtering. Step 2 includes range cell migration correction (RCMC) and range compression. Step 3 implements partial dechirp. Azimuth compensation is accomplished in Step 4.

\subsection{Operations in the Range Direction}

After transforming Equation (1) into the range frequency domain, the return signal is given as:

$$
S_{r n g F}\left(t, f_{\tau} ; t_{c}, \Delta R\right)=\sigma \cdot \operatorname{rect}\left(\frac{t-t_{c}}{T_{s}}\right) \cdot \operatorname{rect}\left(\frac{f_{\tau}}{B_{r}}\right) \cdot \exp \left(-j \pi \frac{f_{\tau}^{2}}{K_{r}}\right) \cdot \exp \left[-j \frac{4 \pi\left(f_{\tau}+f_{0}\right)}{c} R(t)\right],
$$

where $f_{\tau}$ is the range frequency, $B_{r}=K_{r} T_{p}$ is the bandwidth of the transmitted signal, and $f_{0}$ is the carrier frequency. To increase the accuracy of RCMC, the term $k_{1,1, r} \cdot \Delta R$ in Equation (14) should first be removed. Because $k_{1,1, r} \cdot \Delta R$ depends on the position of a target, the return signal must be segmented in the range direction. The length of each segment $\Delta R_{\text {seg }}$ can be 1.5-2 times $\Delta R_{B}$. Then, the range-dependent term $k_{1,1, r} \cdot \Delta R$ can be compensated in each segment by the following first-order filter:

$$
H_{\text {Lrcmc }}=\exp \left\{j \frac{4 \pi\left(f_{0}+f_{\tau}\right)}{c} \cdot k_{1,1, r} \cdot \Delta R_{\text {seg }} \cdot t\right\} .
$$

Subsequently, RCMC and range compression are implemented using the fourth-order nonlinear chirp scaling imaging algorithm [22], which extends the RCMC ability in the range direction, and the echo becomes (Appendix A):

$$
S\left(t, \tau ; t_{c}, \Delta R\right)=\sigma \cdot \operatorname{rect}\left(\frac{t-t_{c}}{T_{s}}\right) \cdot \sin c\left(\tau-\tau_{\text {mig }}\right) \cdot \exp \left\{-j \frac{4 \pi}{\lambda} R(t)\right\},
$$

where $\tau_{m i g}=2 R_{c} / c-T_{S_{-} R W C}$. In addition, $R(t)$ has the same form as that given in Equation (2), where $r_{1}=k_{1,1, a} \cdot \Delta t_{c}$, and all other parameters, including $r_{0}, r_{2}, r_{3}, r_{4}$, and $r_{5}$, are the same as those given in Equation (3). 


\subsection{Operation in the Azimuth Direction}

Because the signal has been well compressed in the range direction, the azimuth operations can be implemented in each range gate. The first time-scaling given as:

$$
H_{A S 1}(t ; \Delta R)=\exp \left\{\left.j \frac{2 \pi}{\lambda} k_{1,1, a}\right|_{\Delta R} t^{2}\right\}
$$

is used to remove the azimuth-dependent term $\left.k_{1,1, a}\right|_{\Delta R} \cdot \Delta t_{c}$. Then, the frequency-scaling

$$
H_{Y_{3} Y_{4}}\left(f_{t} ; \Delta R\right)=\exp \left\{j \frac{\left.2 \pi Y_{3}\right|_{\Delta R}}{3 \lambda}\left(\frac{\lambda}{2}\right)^{3} f_{t}^{3}-j \frac{\left.\pi Y_{4}\right|_{\Delta R}}{\lambda}\left(\frac{\lambda}{2}\right)^{4} f_{t}^{4}\right\}
$$

and the second time-scaling

$$
H_{A S 2}(t)=\exp \left\{-j \frac{4 \pi}{\lambda} \cdot \sum_{n=3}^{5} \frac{p_{n}}{n !} t^{n}\right\}
$$

are subsequently applied to correct the quadratic and linear spatial variances in $r_{n}(n=3,4,5)$, respectively. Expressions for the undefined parameters in Equations (19) and (20), including $Y_{3}, Y_{4}, p_{3}$, $p_{4}$, and $p_{5}$, are given in Appendix B. After removing the azimuth spatial variance in $r_{n}(n=3,4,5)$, the higher-order items in the frequency domain can be compensated by applying

$$
H_{a p c}\left(f_{t}\right)=\exp \left\{-j \frac{4 \pi}{\lambda} \sum_{m=3}^{10} P_{m}\left(\frac{\lambda f_{t}}{2}\right)^{m}\right\},
$$

where the values of $P_{m}(m=3-10)$ are defined in Appendix C. Implementing Equations (18)-(21) transforms the return signal as follows:

$$
\begin{aligned}
S_{a p c}\left(t, \tau ; t_{c}, \Delta R\right) \approx & \sigma \cdot \operatorname{rect}\left(\frac{t-t_{c}}{T_{s}}\right) \cdot \sin c\left(\tau-\tau_{m i g}\right) \\
& \cdot \exp \left\{-j \frac{4 \pi}{\lambda}\left[r_{0}-\frac{\left.k_{1,1, a}\right|_{\Delta R}}{2} t_{c}^{2}+\frac{1}{2}\left(r_{2}-\left.k_{1,1, a}\right|_{\Delta R}\right)\left(t-t_{c}\right)^{2}+\sum_{n=3}^{5} \frac{\left.r_{n}\right|_{\Delta t_{c}=0}}{n}\left(t-t_{c}\right)^{n}\right]\right\}
\end{aligned}
$$

The time-frequency relationships corresponding to Equations (17) and (22) are illustrated in Figure 11a,b, respectively. Here, the Doppler bandwidth is transformed from $B_{a_{-} \text {origin }} \approx 2\left|r_{2}\right| T_{s} / \lambda$ to $B_{a_{-} A S} \approx 2\left|r_{2}-k_{1,1, a}\right|_{\Delta R} \mid T_{S} / \lambda$. However, the Doppler bandwidth must be recovered to preserve the azimuth resolution. This is implemented using partial dechirp, which consists of applying the following two steps:

$$
\left\{\begin{array}{l}
H_{P D_{-} 1}\left(f_{t}\right)=\exp \left\{-j 2 \pi \cdot \chi \cdot \phi_{0}\left(f_{t}\right)\right\} \\
H_{P D_{-} 2}(t)=\exp \left\{-j \frac{4 \pi}{\lambda} \cdot \frac{\left.k_{1,1,1}\right|_{\Delta R}}{2(1-\chi)} t^{2}\right\}
\end{array}\right.
$$

Here, $\phi_{0}\left(f_{t}\right)$ can be calculated according to Equation (A8) in Appendix A, and $\chi$ is the partial dechirp coefficient, where $0<\chi<1$. The application of Equation (23) transforms the echo as follows:

$$
\begin{aligned}
S_{P D}\left(t, \tau ; t_{c}, \Delta R\right)= & \sigma \cdot \operatorname{rect}\left[\frac{t-t_{c}}{(1-\chi) T_{s}}\right] \cdot \sin c\left(\tau-\tau_{m i g}\right) \\
& \cdot \exp \left\{-j \frac{4 \pi}{\lambda}\left[r^{\prime}{ }_{0}+r^{\prime}{ }_{1} \cdot\left(t-t_{c}\right)+\frac{r^{\prime} 2}{2} \cdot\left(t-t_{c}\right)^{2}+\sum_{n=3}^{5} \frac{\left.r_{n, r e f}\right|_{\Delta R}}{n}\left(t-t_{c}\right)^{n}\right]\right\}
\end{aligned}
$$

where $r_{0}^{\prime}=r_{0}+\left.k_{1,1, a}\right|_{\Delta R} \cdot[1 /(1-\chi)-1 / 2], r_{1}^{\prime}=\left.2 k_{1,1, a}\right|_{\Delta R} \cdot t_{c} /(1-\chi)$, and $r_{2}^{\prime}=r_{2} /(1-\chi)$. The azimuth time-frequency relationship corresponding to Equation (24) is shown in Figure 11c. The Doppler bandwidth is recovered and the span of the aperture time is reduced to $T_{s_{-} p d}=(1-\chi) T_{s}$. 
(a)

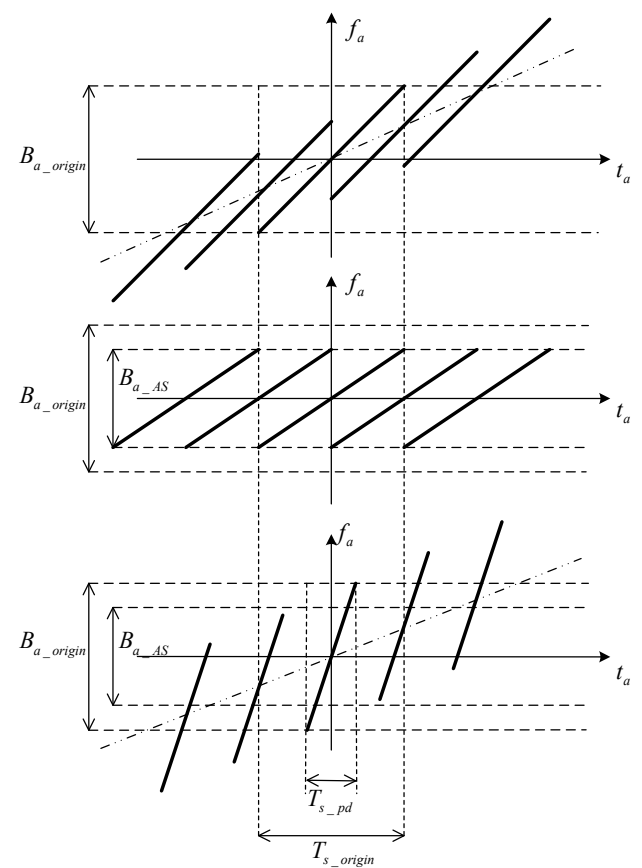

Figure 11. Azimuth time-frequency relationships corresponding to Equations (17), (22), and (24) are illustrated in $(\mathbf{a}-\mathbf{c})$, respectively.

After conducting partial dechirp, azimuthal segmentation is employed, where the width of each segment is $(1-\chi) T_{s}$. We can compensate for the azimuth-dependent term in Equation (24) by applying

$$
H_{\text {subscene }}\left(t ; t_{c, \text { sub }}\right)=\exp \left\{j \frac{4 \pi}{\lambda} \frac{\left.k_{1,1, a}\right|_{\Delta R} t_{c, s u b}}{(1-\chi)}\left(t-t_{c, s u b}\right)\right\}
$$

to each azimuth segment, where $t_{c, s u b}$ denotes the center time of each azimuth segment. Once the spatial variance in the azimuth direction has been removed, azimuth compression is implemented by applying:

$$
H_{\text {aziCompress }}\left(f_{t}\right)=\exp \left\{-j \frac{\pi \lambda(1-\chi)}{\left.2 r_{2}\right|_{\Delta t_{c}=0}} f_{t}^{2}\right\} .
$$

Then, the final imaging result is obtained by transforming the return signal into the $2 \mathrm{D}$ time domain.

\section{Simulation and Analysis}

\subsection{Simulation Method and Parameters}

Since the flight speed of GEO SAR is nearly equivalent to the earth's rotation, the relative speed between targets and the satellite drastically vary with the satellite's position, termed the argument of latitude (AOL). This varies the slant range history in different azimuth time, i.e., the Doppler characteristic is spatially variant. Only one tailored frequency-domain imaging algorithm cannot focus well in the full orbit time. In order to evaluate the feasibility over the full orbit time, the spatial variance condition of the echoes should be quantized. Measuring the variance involves describing the relationship between the linear and nonlinear component of the Doppler signal in a single synthetic aperture time. The higher linear proportion means that the echo is less spatial, and vice versa. According to the relationship between the slant range and Doppler, the maximum linear component in one synthetic aperture time is $\left|r_{2}\right| T_{s} / 2$ according to Equation (2). The maximum nonlinear part is 
$\left|r_{3}\right| T_{s}^{2} / 8$. Due to $r_{2}$ potentially being zero in some AOL positions, we mapped the ratio between these two into a negative exponential function, and introduced the spatial variance index:

$$
g_{\mathrm{var}}=\exp \left\{-\frac{\left|r_{3}\right|}{\left|r_{2}\right|} \cdot \frac{T_{s}}{4}\right\}
$$

This means that when $g_{v a r}$ is closer to 1 , the linear component accounts for a larger proportion, and then the spatial variance weakens, and vice versa.

Another key factor dominating the performance of an imaging algorithm is the squint angle. As mentioned before, the squint angle is much smaller in GEO SAR, limited to $\pm 8^{\circ}$, than in LEO SAR. This means that the traditional squint angle, or together with the elevation angle, cannot describe the squint characteristic in GEO SAR. Therefore, the ground squint angle was introduced to evaluate the squint condition in GEO SAR [18], which is the projection angle on ground of the traditional squint angle. The relationship of these angles is demonstrated in Figure 12.

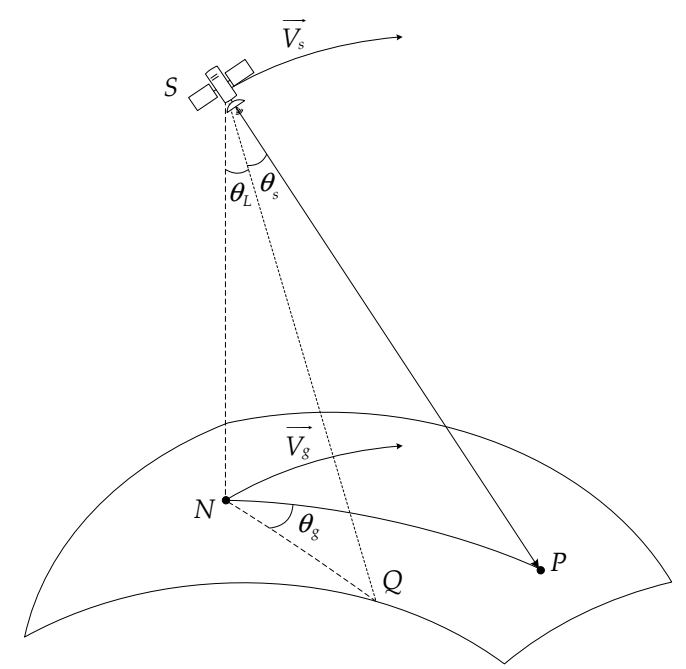

Figure 12. The observation angle relationships in GEO SAR. $S$ denotes the satellite position, $P$ is the target position on the ground, and $N$ is the nadir point. SP is the beam-center line. SNQ is the zero-Doppler plane, $S N P$ is the squint plane, $\theta_{L}$ is the elevation angle, $\theta_{S}$ is the squint angle, and $\theta_{\mathrm{g}}$ is the ground squint angle.

The simulation parameters are listed in Table 1. The orbital parameters refer to the Global Earthquake Satellite System (GESS) projection [3]. The swath center is selected as Wenchuan County in China, which is a highly seismic zone requiring frequent observation.

Table 1. Simulation parameters.

\begin{tabular}{cc}
\hline Parameter & Value \\
\hline Semi-major Axis $(\mathrm{km})$ & $42,164.3$ \\
Inclination $\left(^{\circ}\right)$ & 60 \\
Eccentricity & 0 \\
Longitude of Ascend Node $\left(^{\circ}\right)$ & 100 \\
Wavelength $(\mathrm{m})$ & 0.24 \\
Longitude of Wenchuan County, China $\left(^{\circ}\right)$ & $103.4 \mathrm{E}$ \\
Latitude of Wenchuan County, China $\left(^{\circ}\right)$ & $31.0 \mathrm{~N}$ \\
\hline
\end{tabular}

\subsection{Observation Duration Comparison}

Based on the simulation parameters listed in Table 1, the full orbit observations by broadside and squint modes were simulated, and the results are shown in Figure 13. Here, the blue lines represent 
the nadir trajectory, the red lines represent the feasible observation durations corresponding to the respective observation modes, and the green point represents the target location.

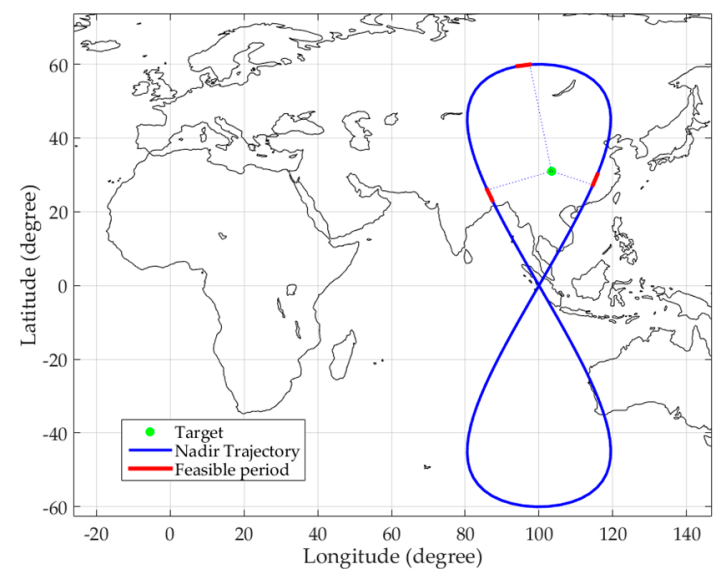

(a)

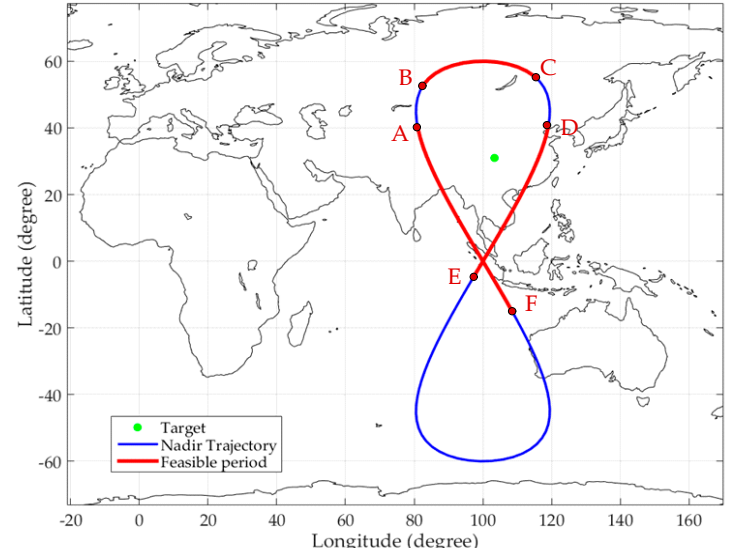

(b)

Figure 13. Comparison of GEO SAR observation durations obtained for a nadir trajectory mode (a) in broadside and (b) the proposed squint mode method.

For the broadside mode, there are only three positions that can be used to observe Wenchuan, as shown in Figure 13a. The AOLs of three positions are nearly $30^{\circ}, 87^{\circ}$, and $148^{\circ}$. At these positions, the squint angle and elevation angle, denoted by $\left(\theta_{L}, \theta_{s}\right)$, should be $\left(1.92^{\circ}, 2.10^{\circ}\right),\left(4.82^{\circ}, 0.51^{\circ}\right)$, and $\left(0.99^{\circ},-1.56^{\circ}\right)$. The ground squint angles of these three positions are equal to zero. Applying the broadside mode yields an observation duration of about $0.59 \mathrm{~h}$ per orbit, which is $2.47 \%$ of the orbital period. In contrast, through adjusting the observing angles in different AOLs, the proposed algorithm can image Wenchuan for several strip areas, as shown in Figure 13b. Applying the proposed method, the observation duration increased to about $11.55 \mathrm{~h}$ per orbit, which is $48.15 \%$ of the orbital period. The results indicate that the proposed method significantly improves the observation ability of GEO SAR.

\subsection{Imaging Results and Analysis}

Figure 13 shows the feasible observation period in different modes. There is no need to clarify the feasible period in Figure 12a because so many broadside imaging algorithms have that ability. However, the feasible periods in Figure $12 \mathrm{~b}$ were created by the proposed algorithm, which should be verified. In Figure 13b, three sections, $F A, B C$, and $D E$, are stated as the feasible periods. According to the simulation parameters listed in Table 1 , we drew the spatial variance index $g_{v a r}$ and ground squint angle $\theta_{g}$ curves in the full orbit, which are shown in Figure 14.

According to Section 5.1, the most difficult position for imaging are among A F. Therefore, the main characteristics of these points are listed in Table 2.

According to Table 2, position A has the most severe spatial variance $\left(g_{v a r}=0.955\right)$, and position $\mathrm{D}$ has the largest ground squint angle $\left(\theta_{g}=62.53^{\circ}\right)$. Hence, if the imaging qualification in $\mathrm{A}$ and $\mathrm{D}$ can be guaranteed, then all the other positions in the feasible period in Figure 13b can be focused well.

We employed the simulated scene illustrated in Figure 15 covering an area of $30 \times 30 \mathrm{~km}$ to verify the imaging performance of the proposed method. The scene includes three point targets, $T_{1}, T_{2}$, and $T_{3}$, arranged at the lower left corner, the center, and the upper right corner, respectively. Two group experiments were carried out. The first was at position A. The algorithm designed by Zhanget al. [18] and the proposed algorithm were compared. The other experiment was at position $\mathrm{D}$, and only the results by the proposed algorithm are shown. All other parameters were the same as those listed in Tables 1 and 2. 


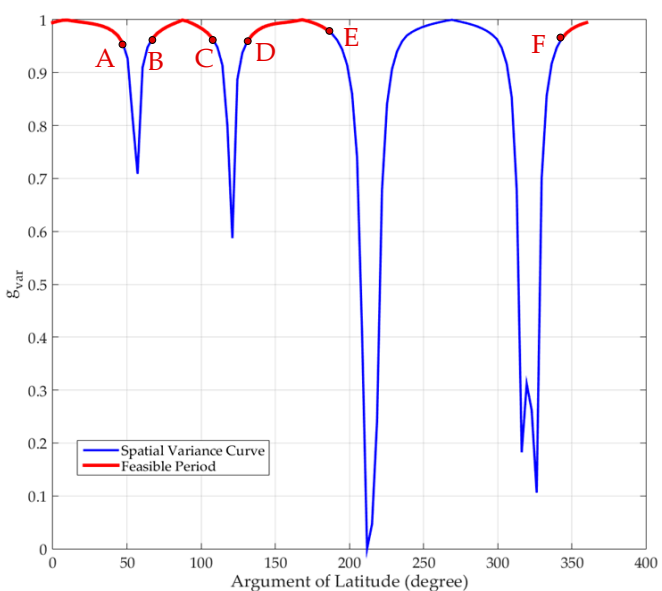

(a)

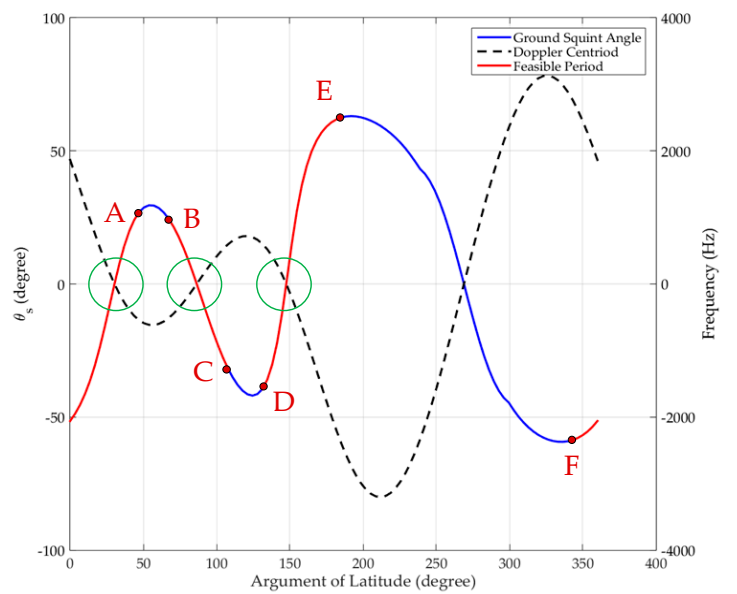

(b)

Figure 14. The spatial variance index and ground squint angle curves when observing Wenchuan in full orbit. (a) The spatial variance index $g_{\text {var }}$; (b) the ground squint angle $\theta_{g}$. The black dashed line is the Doppler centroid frequency, which has three intersection points with the ground squint angle curve at $\theta_{g}=0$. These three points are highlighted by green circles, corresponding to the broadside mode in Figure 13a.

Table 2. Characteristics of candidate positions.

\begin{tabular}{cccccccc}
\hline Index & $\left.\operatorname{AOL~}^{(}\right)$ & Longitute-Latitude of Nadir $\left(^{\circ}\right)$ & $g_{v a r}$ & $\theta_{g}\left({ }^{\circ}\right)$ & $\theta_{L}\left({ }^{\circ}\right)$ & $\theta_{s}\left({ }^{\circ}\right)$ & $T_{s}(\mathbf{s})$ \\
\hline A & 47.10 & $(81.18 \mathrm{E}, 39.38 \mathrm{~N})$ & $\mathbf{0 . 9 5 5}$ & 26.95 & 3.18 & 1.30 & 365.08 \\
\hline $\mathrm{B}$ & 67.29 & $(82.78 \mathrm{E}, 53.02 \mathrm{~N})$ & 0.965 & 24.48 & 4.43 & 0.67 & 465.53 \\
\hline $\mathrm{C}$ & 107.66 & $(114.83 \mathrm{E}, 55.61 \mathrm{~N})$ & 0.965 & -31.66 & 4.35 & 0.37 & 529.99 \\
\hline $\mathrm{D}$ & 131.22 & $(119.07 \mathrm{E}, 40.65 \mathrm{~N})$ & 0.959 & -39.51 & 2.74 & -0.43 & 428.13 \\
\hline $\mathrm{E}$ & 185.05 & $(97.48 \mathrm{E}, 4.37 \mathrm{~S})$ & 0.981 & $\mathbf{6 2 . 5 3}$ & -3.61 & -4.48 & 714.52 \\
\hline F & 343.18 & $(108.23 \mathrm{E}, 14.51 \mathrm{~S})$ & 0.964 & -58.63 & -4.15 & 5.52 & 722.26 \\
\hline
\end{tabular}

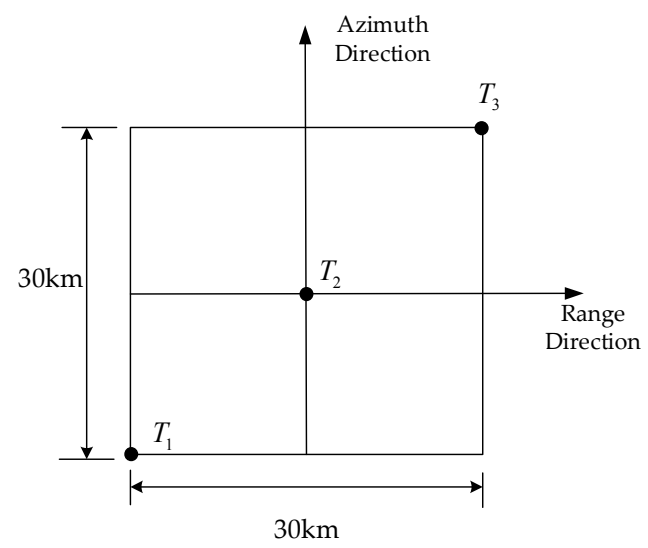

Figure 15. Simulation scene covering an area of $30 \times 30 \mathrm{~km}$, where point $T_{2}$ is at the swath center.

At position A, the imaging results for the simulated scene given in Figure 15 obtained by the 2D nonlinear CS algorithm [18] and the proposed algorithm are presented in Figures 16 and 17, respectively. Here, the first, second, and third rows correspond to points $T_{1}, T_{2}$, and $T_{3}$ illustrated in Figure 15, respectively. The first, second, and third columns represent contour maps, azimuth profiles, and range profiles, respectively. In both figures, contour maps denote the $2 \mathrm{D}$ focusing quality, and the profiles represent the focusing quality along the range and azimuth directions. The data given in Figures 16 
and 17 were evaluated according to resolution (res), peak to side lobe ratio (PSLR), and integrated side lobe ratio (ISLR) along the azimuth and range directions, and the results are presented in Table 3 . Here, PSLR is defined as the ratio of the peak level of sidelobes to the peak level of the main lobe, and the ISLR is the ratio of the total power in all sidelobes to the power in the main lobe.

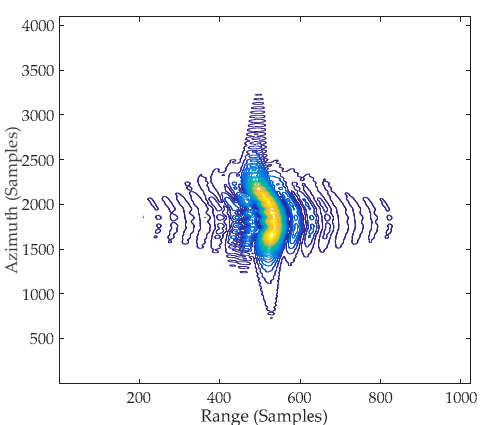

(a)

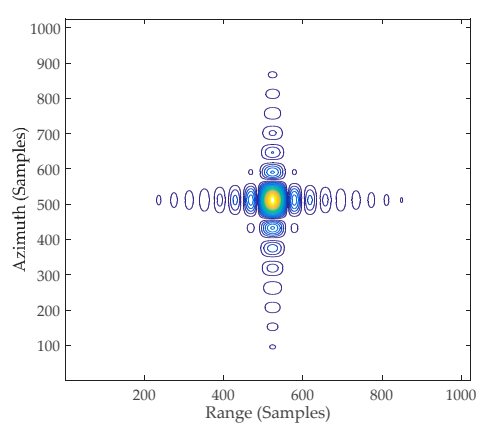

(d)

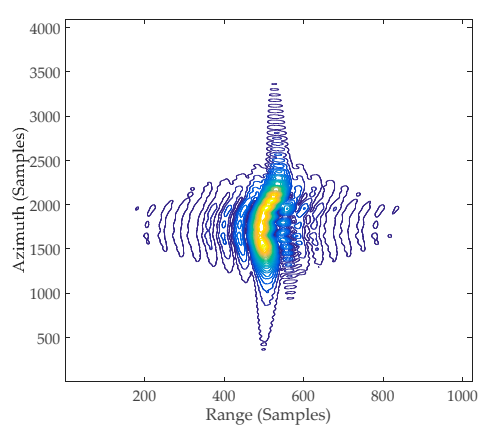

(g)

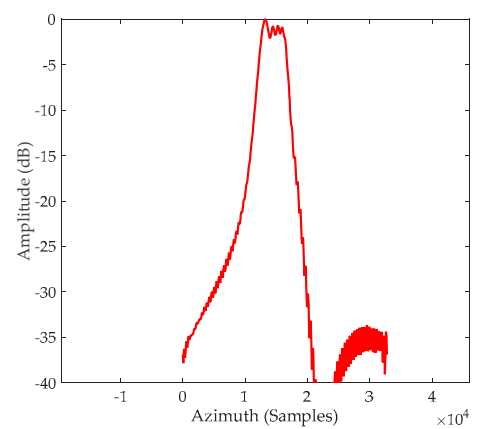

(b)

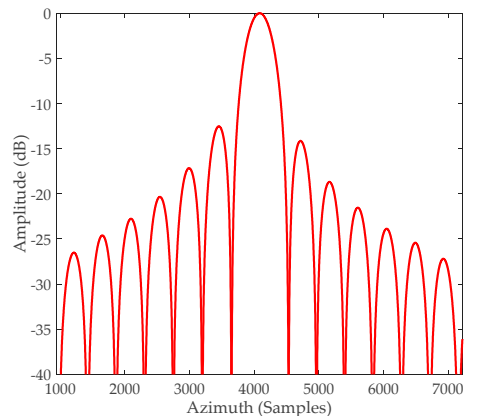

(e)

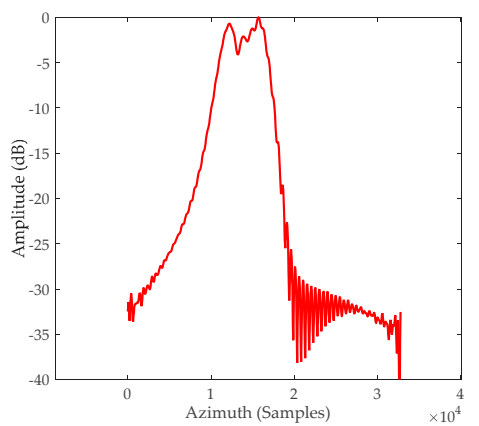

(h)

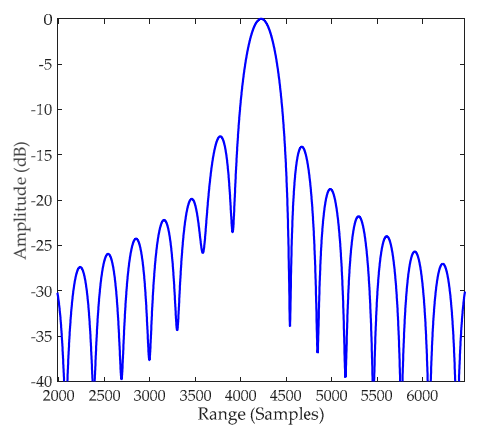

(c)

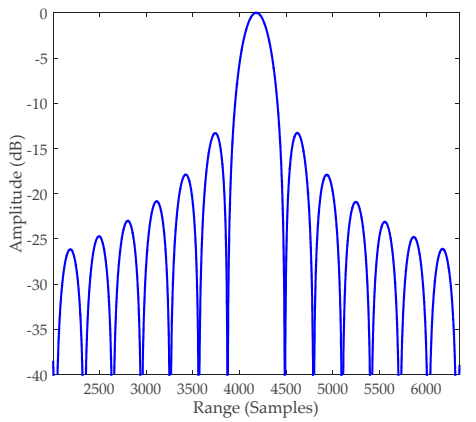

(f)

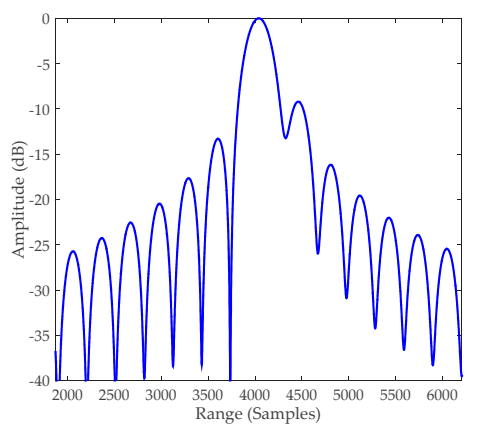

(i)

Figure 16. Imaging results of the $2 \mathrm{D}$ nonlinear chirp scaling (CS) algorithm [18]. The first (a-c), second (d-f), and third (g-i) row correspond respectively to points $T_{1}, T_{2}$, and $T_{3}$ illustrated in Figure 15. The first $(\mathbf{a}, \mathbf{d}, \mathbf{g})$, second $(\mathbf{b}, \mathbf{e}, \mathbf{h})$, and third $(\mathbf{c}, \mathbf{f}, \mathbf{i})$ column represent contour maps, azimuth profiles, and range profiles, respectively.

Table 3. Evaluation of imaging quality (in position A).

\begin{tabular}{cccccccc}
\hline & \multicolumn{4}{c}{ Azimuth Direction } & \multicolumn{3}{c}{ Range Direction } \\
\hline Method & Target & Res (m) & PSLR (dB) & ISLR (dB) & Res (m) & PSLR (dB) & ISLR (dB) \\
\hline Algorithm & $\mathrm{T}_{1}$ & 55.622 & -15.129 & -19.633 & 1.578 & -12.963 & -10.624 \\
proposed by & $\mathrm{T}_{2}$ & 4.810 & -12.525 & -10.279 & 1.522 & -13.279 & -10.050 \\
Zhang et al. [18] & $\mathrm{T}_{3}$ & 33.486 & -0.809 & -1.828 & 1.522 & -9.213 & -7.721 \\
\hline \multirow{2}{*}{ Proposed } & $\mathrm{T}_{1}$ & 4.775 & -13.256 & -10.295 & 1.534 & -13.351 & -10.138 \\
Algorithm & $\mathrm{T}_{2}$ & 4.800 & -13.168 & -10.153 & 1.534 & -13.264 & -10.039 \\
& $\mathrm{~T}_{3}$ & 4.973 & -13.323 & -10.091 & 1.534 & -13.310 & -10.119 \\
\hline
\end{tabular}




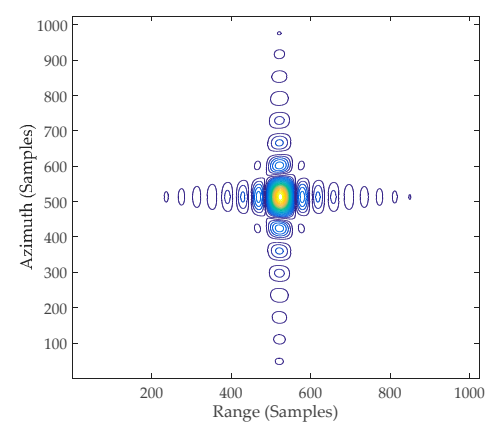

(a)

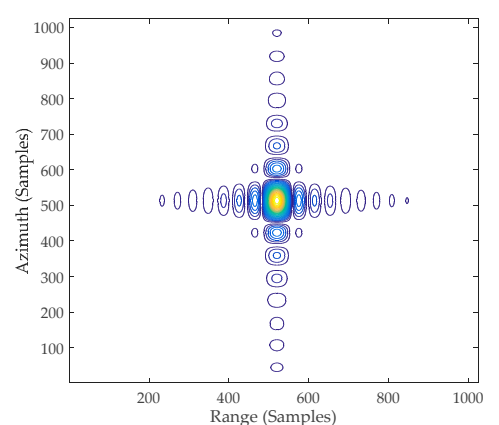

(d)

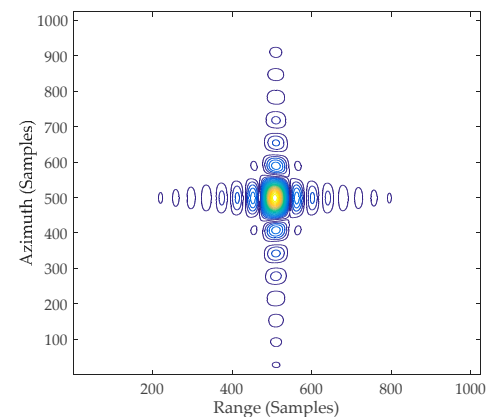

(g)

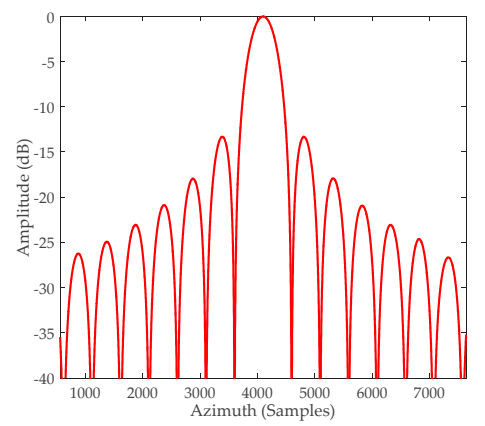

(b)

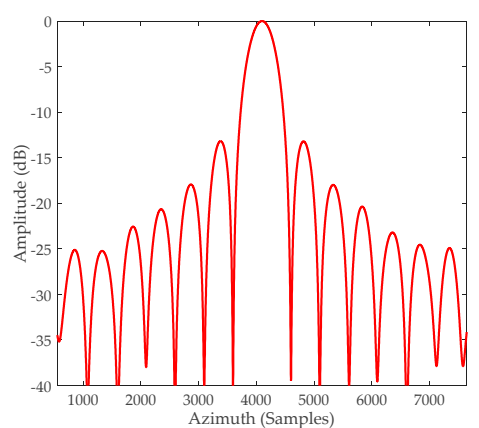

(e)

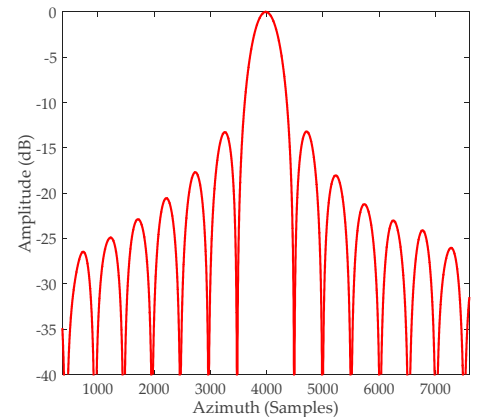

(h)

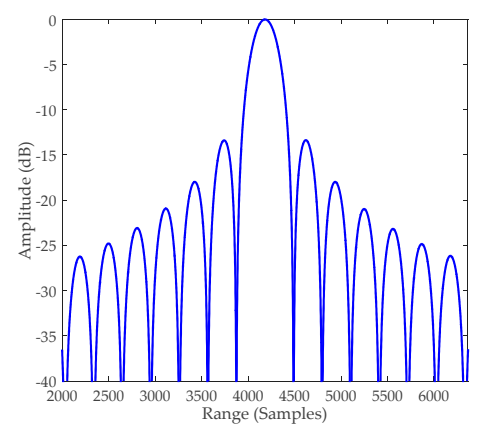

(c)

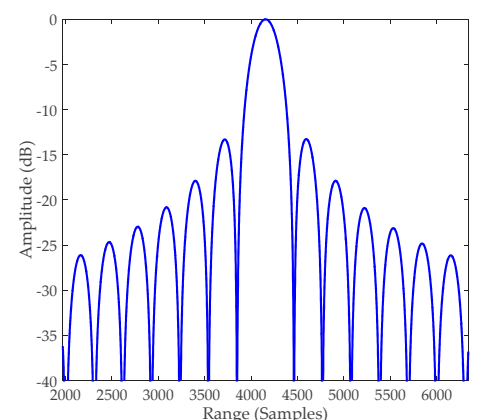

$(\mathbf{f})$

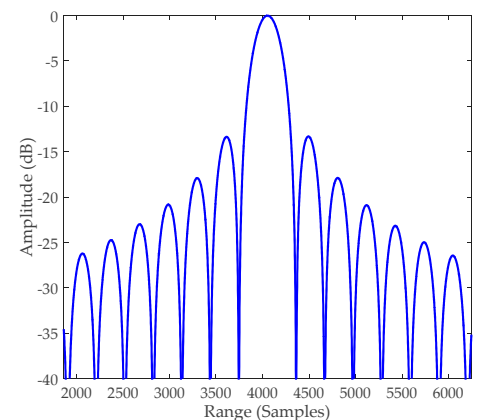

(i)

Figure 17. Imaging results of the proposed squint mode algorithm. Images (a-i) are equivalent representations to those given in Figure 16.

The algorithm proposed by Zhang et al. [18] neglects the residual walk after removing the bulk range walk, and this negatively affects the azimuth spectrum of the imaging results, as indicated by Figures 16 and 17 and Table 3. This issue becomes increasingly serious as the spatial variance increases. As shown in Figure 16, the swath center point $T_{2}$ is well focused. However, for points $T_{1}$ and $T_{3}$, which are at the edges of the swath, the signal in azimuth is almost defocused. Since the spatial variance is so severe that the traditional nonlinear chirp scaling does not work well, the imaging quality in range direction is degraded. However, the proposed algorithm preserves the focusing performance of the 2D nonlinear CS algorithm over the full scene. From the quality indices presented in Table 3, the imaging coherency is good over the entire swath. Since the fourth-order nonlinear chirp scaling is used in range direction, the focusing ability in range direction is improved compared to the algorithm proposed by Zhang et al. [18].

Position D has the largest ground squint angle, which should be simulated to verify the performance of proposed imaging algorithm. Since the performance comparison with the start-of-art algorithm has been presented in position A above, only the results of the proposed algorithm are shown here, in Figure 18 and Table 4. The simulation parameters are listed in Tables 1 and 2, and the simulation layout is the same as shown in Figure 15. 


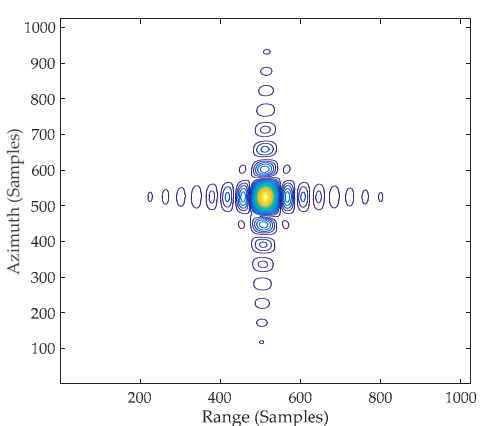

(a)

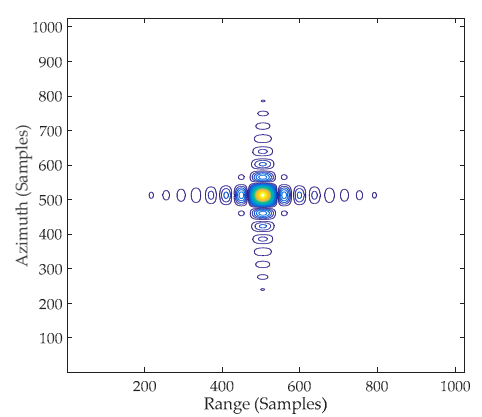

(d)

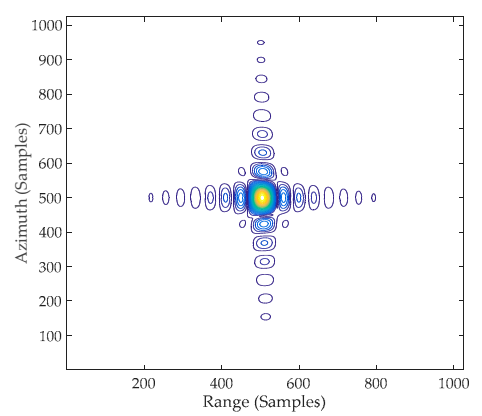

(g)

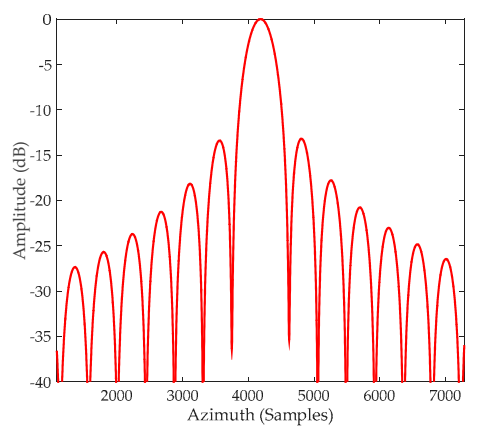

(b)

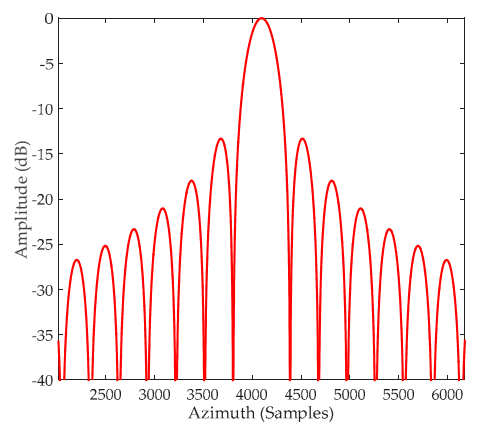

(e)

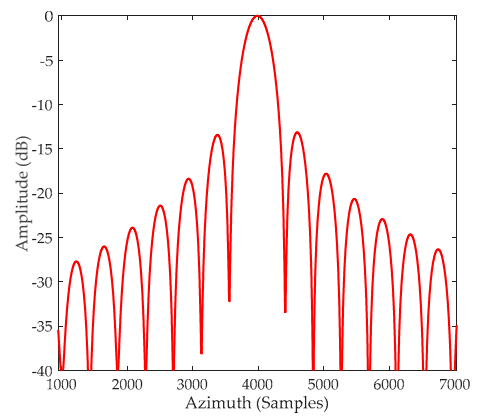

(h)

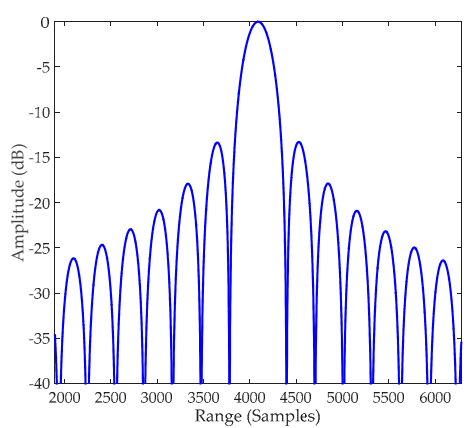

(c)

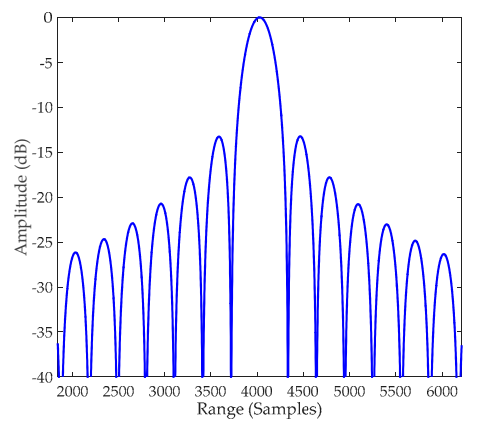

(f)

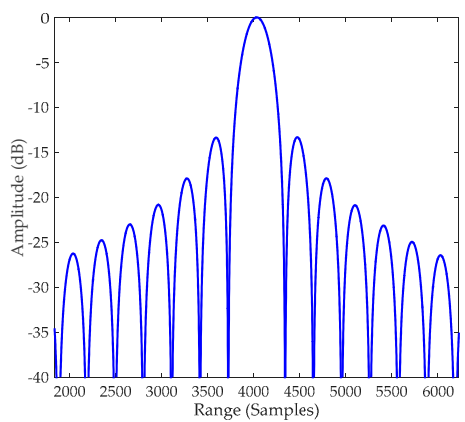

(i)

Figure 18. Imaging results of the proposed squint mode algorithm. Images (a-i) are equivalent representations to those given in Figure 16.

Table 4. Evaluation of imaging quality (in position D).

\begin{tabular}{cccccccc}
\hline & & \multicolumn{3}{c}{ Azimuth Direction } & \multicolumn{3}{c}{ Range Direction } \\
\hline \multirow{2}{*}{ Method } & Target & Res (m) & PSLR (dB) & ISLR (dB) & Res (m) & PSLR (dB) & ISLR (dB) \\
\hline \multirow{2}{*}{ Proposed } & T1 & 4.656 & -13.233 & -9.695 & 1.318 & -13.298 & -10.097 \\
Algorithm & T2 & 4.866 & -13.310 & -10.253 & 1.309 & -13.233 & -10.031 \\
& T3 & 4.952 & -13.158 & -10.250 & 1.318 & -13.324 & -10.139 \\
\hline
\end{tabular}

The simulation results shown in Figure 18 and Table 4 together verify the imaging performance in the most severe squint looking conditions. It is obvious that our method has good imaging ability over the full scene. However, the focusing effect in the azimuth direction is little degraded compared to position A, as shown in the azimuth profiles in Figure 18. However, this performance degradation is acceptable for most applications. 


\section{Discussion}

\subsection{Accuracy}

In order to correct the squint effect, the linear residual terms $k_{1,1, r} \cdot \Delta R$ and $\left.k_{1,1, a}\right|_{\Delta R} \cdot \Delta t_{c}$ in Equation (3) were removed since these two terms depend on the locations of targets. Segmentation was adopted by applying Equations (16) and (25). After segmentation, each sub-block corresponds to an area of $\Delta R_{\text {seg }} \times(1-\chi) T_{s}$, as shown in Figure 19. When processing each sub-block of data, the follow-up filters were designed according to the center point $P_{C}$. Therefore, for all points except $P_{C}$, residual phase errors occur, which have to be controlled to guarantee the imaging accuracy.

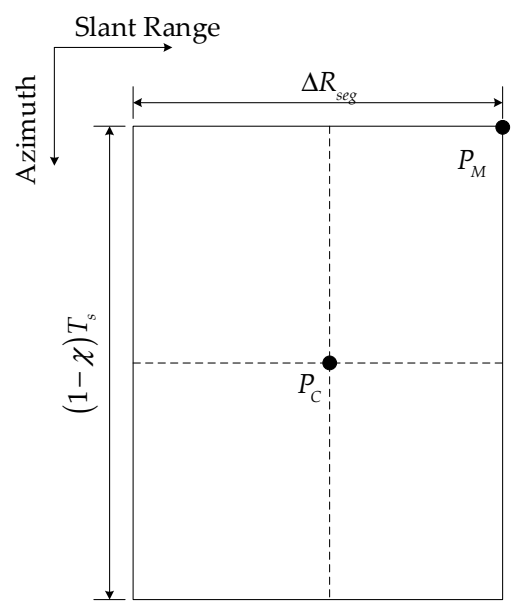

Figure 19. Points in one sub-block diagram. Point $P_{C}$ is located at the sub-block center, whereas $P_{M}$ is a set denoting every point at the corner.

Suppose a target located at $(\Delta t, \Delta R)$ has an accurate 2D frequency domain phase $\Phi^{a}(\Delta t, \Delta R)$ and the polynomial expansion phase $\Phi^{e}(\Delta t, \Delta R)$. In some positions where the spatial variance is minimal, the range walk removed echo data are like broadside data, which means $r_{1} \approx 0$ and $\Phi^{a} \approx \Phi^{e}$. However, for some severe spatial variant areas, $k_{1,1, r} \cdot \Delta R$ and $\left.k_{1,1, a}\right|_{\Delta R} \cdot \Delta t_{c}$ change drastically. The expansion $\Phi^{e}(\Delta t, \Delta R)$ is no longer accurate. Segmentation can be used to correct it. Therefore, the correct approach to weigh the segmentation is to improve the quality of the phase error of the corner points. That is,

$$
\Delta \Phi\left(\chi, \Delta R_{s e g} / \Delta R_{B}\right)=\max _{P \in P_{M}}\left|\Phi_{P}^{e}\left(\Delta t \pm \frac{T_{s}}{2}(1-\chi), \Delta R \pm \frac{1}{2} \Delta R_{s e g}\right)-\Phi_{P}^{a}\left(\Delta t \pm \frac{T_{s}}{2}(1-\chi), \Delta R \pm \frac{1}{2} \Delta R_{s e g}\right)\right|
$$

where

$$
\left\{\begin{array}{l}
\Phi^{a}(\Delta t, \Delta R)=-\frac{4 \pi\left(f_{\tau}+f_{0}\right)}{c}\left[r_{0}-\sum_{n=1}^{9} \frac{A_{n}}{n+1}\left(-\frac{c f_{t}}{2\left(f_{0}+f_{\tau}\right)}-r_{1}\right)^{n+1}\right] \\
\Phi^{e}(\Delta t, \Delta R)=\Phi\left(f_{t}, f_{\tau}\right)
\end{array},\right.
$$

and the $\Delta t$ and $\Delta R$ are inherent in $r_{n}$ and $A_{n}$, which are defined in Equations (3) and (A9). $\Phi\left(f_{t}, f_{\tau}\right)$ is shown in Equation (A7).

The relative phase $\Delta \Phi$ should be controlled at a very low level, which means segmentation contributes to an accurate expression without introducing phase error. To this end, Equation (28) should obey

$$
\Delta \Phi\left(\chi, \Delta R_{s e g} / \Delta R_{B}\right)<\frac{\pi}{4 M^{\prime}}
$$

where $M>1$ is an adjustable coefficient.

Based on this criterion, parameters $\Delta R_{\text {seg }}$ and $\chi$ can be selected to ensure the residual parts after segmenting are small enough for subsequent operations. It also shows that segmentation does not 
introduce error by this means. Using the simulation examples demonstrated in Section 5 , the simulated relationship among $\Delta R_{\text {seg }}, \chi$, and $\Delta \Phi$ is demonstrated in Figure 20.

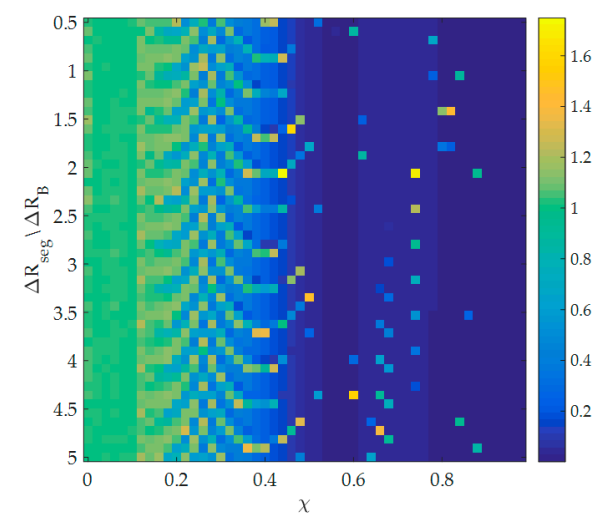

(a)

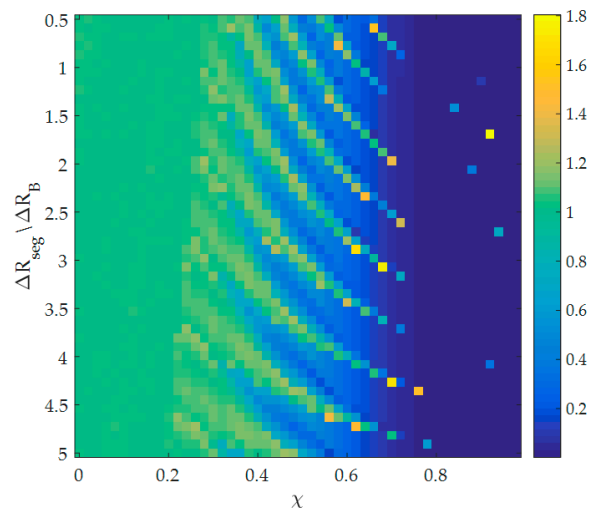

(b)

Figure 20. The simulated relative phase $\Delta \Phi$ against $\chi$ and $\Delta R_{\text {seg. }}$ (a) and (b) correspond to targets $T_{3}$ in position $\mathrm{A}$ and position $\mathrm{D}$ in Table 2, respectively. These two images reflect the corner points' $P_{M}$ phase error of controlled by $\Delta R_{\text {seg }}$ and $\chi . \Delta \Phi$ heavily relies on $\chi$, which means the azimuth spatial variance is more severe than in the range direction. This was verified by imaging results in Figure $16 \mathrm{~g}, \mathrm{~h}$. From the simulation results, $\chi$ should be larger than 0.8 , whereas $\Delta R_{\text {seg }}$ can be several times $\Delta R_{B}$ with high $\Delta \Phi$ values avoided.

The final imaging quality does not depend on Equation (30); instead, quality depends on the fourth-order NCS in range direction and time-frequency scaling in the azimuth direction. Equation (30) only ensures that segmentation has no effect on the subsequent image quality. Since the fourth-order NCS and time-frequency are designed for imaging a broad scene, the imaging quality of targets in small $30 \times 30 \mathrm{~km}$ areas can be ensured in most circumstances. This means that the marginal points of sub-blocks can be focused. The error between sub-blocks can only be distorted, which can be resolved by geometric calibration before stitching.

\subsection{Computational Load}

The computational load is one of the main factors affecting the practical application of the algorithm. To multiply a pair of complex numbers requires six floating point operations per second (FLOPs), whereas only one FLOP is needed for complex addition. Specifically, creating an $N$ points FFT (or IFFT) operation requires $5 N \log _{2}(N)$ FLOPs [20]. Suppose the echo data has $N_{a z i}$ and $N_{r n g}$ sampling points in the azimuth and slant range directions, respectively, after range walk removal. For the BP algorithm, with eight-times interpolation in the range direction, and imaging an area of $N_{r n g} \times N_{r n g}$ size, the computational load of $\mathrm{BP}$ is:

$$
C_{B P}=45 N_{a z i} N_{r n g} \log _{2}\left(N_{r n g}\right)+7 N_{a z i} N_{r n g}^{2} .
$$

However, for the algorithm proposed, there are $4 N_{a z i}$ times $N_{r n g}$ points FFT operations, $10 N_{r n g}$ times $N_{a z i}$ points FFT operations, and $14 N_{a z i} N_{r n g}$ times multiplications. Therefore, the computational load is:

$$
C_{p} \approx 20 N_{a z i} N_{r n g} \log _{2}\left(N_{r n g}\right)+50 N_{a z i} N_{r n g} \log _{2}\left(N_{a z i}\right)+84 N_{a z i} N_{r n g} .
$$

For specific simulation circumstances, the computational load can be numerally determined. Take the simulation circumstance shown in Figure 17 as an example. The azimuth sampling number is 6000 and the slant range sampling number after range removal is 2000. Therefore, the computational load of BP and the proposed algorithm are 173.9 and 11.17 GFLOPs, respectively. The computational burden of the proposed algorithm is only one-fifth that of the BP algorithm, which is suitable 
for practical use. In addition, many segmenting operations exist that can be easily used by parallel computing. This further shortens the time required for the method when implementing in modern computers.

\section{Conclusions}

We proposed a novel squint mode GEO SAR imaging method, where the bulk range walk is first corrected on receive by adjusting the starting time of the receive window and then applying first-order filtering, RCMC, range compression, partial dechirp, and azimuth compression to obtain the focused single looking complex (SLC) image. Simulation results demonstrated that the proposed algorithm achieves a resolution less than $5 \mathrm{~m}$ in the azimuth direction over a $30 \times 30 \mathrm{~km}$ swath.

In addition to the performance, the feasibility of the proposed algorithm was investigated. Firstly, up-to-date technology can make the antenna have the ability to scan in a range of $\pm 8^{\circ}$ in the azimuth direction, which indicates that the staring mode can be realized. Secondly, the low PRF employed with GEO SAR provides ample scope for adjusting the starting time of the receive window and removing the range walk on receive. Lastly, the ephemeris data and geographic information of the target zone can be acquired to calculate the coefficients of the range model and each filter in the proposed algorithm, and focused imaging results can be produced.

The staring mode of GEO SAR enables the continuous observation of the region of interest. Compared with the broadside mode, the proposed method increases the observation duration for Wenchuan County in China by nearly 20 times. As a result, the proposed method enables GEO SAR to conduct long-term observation of target zones, which is essential for the management of global disasters and related affairs. For earthquake-prone areas, like Wenchuan, the starting mode can continuously provide images over a long time period after an earthquake. Some disaster information, including dammed lakes, landslides, and mudslides, can be updated continuously, which is central to disaster relief and rescue.

Another distinctive characteristic of GEO SAR is the short temporal resolution, which is vital to the application of interferometry, such as vegetation measurement and crust deformation detection. However, interferometry cannot be realized by applying several images obtained at different squint angles within one orbit period and coherent change detection needs to use the same observation geometry. For this reason, for interferometric applications, it is necessary to wait at least one orbit period (one day), similarly to the polar orbit repeat pass interferometry case.

Author Contributions: All authors contributed equally to this paper in ideas, design of methodology and writing. Additionally, Z.Y. was responsible for project administration, data analysis and experiments validation, writing. J.G. accomplished software programming, data analysis and presentation.

Funding: This research was funded by China Scholarship Council (Grant No. 201706025038).

Acknowledgments: This work was supported by the Program of the China Scholarship Council under Grant No. 201706025038.

Conflicts of Interest: The authors declare no conflicts of interest.

\section{Appendix A.}

Application of the first-order filter in Equation (16) removes the residual range-Doppler component $k_{1,1, r} \cdot \Delta R$ in Equation (14). Then, Equation (15) is multiplied with the following scaling function:

$$
H_{A S 1}\left(t, f_{\tau}\right)=\exp \left\{-j \frac{4 \pi\left(f_{\tau}+f_{0}\right)}{c} r_{p t}(t)\right\},
$$

where $r_{p t}(t)=-\left.\sum_{n=2}^{4} k_{n, 1, a}\right|_{\Delta R=0} t^{n+1} /(n+1)$ !. Since the observation scene is relatively small, the spatial condition in high-order terms in the frequency domain is also small. That means these parts could be directly compensated for by the reference target's counterparts. In order to improve the RCMC ability in the range direction, the fourth-order nonlinear chirp scaling was introduced [21]. 
Therefore, at this stage, cubic perturbation factor $Y$ and quartic perturbation factor $Z$ were also inserted. Subsequently, the echo was transformed into the 2D frequency domain represented by the azimuth frequency $f_{t}$ and the range frequency $f_{\tau}$, and compensated for by multiplying with:

$$
H_{3 p l u s_{-}} Y\left(f_{t}, f_{\tau}\right)=\exp \left\{-j 2 \pi \sum_{k=3}^{9} \phi_{k, r e f}\left(f_{t}\right) \cdot f_{\tau}^{k}\right\} \cdot \exp \left\{j 2 \pi\left(\frac{1}{3} Y \cdot f_{\tau}^{3}+\frac{1}{4} Z \cdot f_{\tau}^{4}\right)\right\},
$$

where $\phi_{k, r e f}\left(f_{t}\right)$ are the frequency coefficients of the reference target. The expressions for $\phi_{k, r e f}\left(f_{t}\right)$ are provided in Table A1. $Y$ and $Z$ are defined in (A11), at the end of this appendix. According to a previous study [22], the nonlinear CS function is designed as

$$
H_{N C S}\left(f_{t}, \tau\right)=\exp \left\{-j \pi Q_{2}\left(\tau-\tau_{\text {ref }}\right)^{2}-j \frac{2 \pi}{3} Q_{3}\left(\tau-\tau_{\text {ref }}\right)^{3}-j \frac{\pi}{2} Q_{4}\left(\tau-\tau_{r e f}\right)^{4}\right\},
$$

where $\tau_{r e f}$ is the position of the reference target in the range-Doppler domain, and $Q_{2}, Q_{3}$, and $Q_{4}$ are $f_{t}$-dependent scaling coefficients, which are defined in Equation (A11). After conducting the scaling operation, all targets have an equivalent RCM, which can be corrected by multiplying the echo with:

$$
\begin{aligned}
H_{r p c_{-} r c m c}\left(f_{t}, f_{\tau}\right)= & \exp \left\{-j \pi\left[\frac{2}{3} \frac{Y K_{\text {mref }}^{3}+Q_{3}}{\left(K_{\text {mref }}+Q_{2}\right)^{2}} f_{\tau}^{3}+\frac{1}{K_{\text {mref }}+Q_{2}} f_{\tau}^{2}\right]\right\} \\
& \cdot \exp \left\{-j \frac{\pi}{2} \cdot \frac{2\left(J_{\text {mref }}+Q_{3}\right)^{2}-\left(L_{\text {mref }}+Q_{4}\right)\left(K_{\text {mref }}+Q_{2}\right)}{\left(K_{\text {mref }}+Q_{2}\right)^{5}} \cdot f_{\tau}^{4}\right\} \\
& \cdot \exp \left\{j 2 \pi f_{\tau}\left[\phi_{1, \text { ref }}\left(f_{t, \text { ref }}\right)-\phi_{1, \text { ref }}\left(f_{t}\right)\right]\right\}
\end{aligned}
$$

The first and second exponential terms are applied for range compression, whereas the third exponential term is applied for correcting the bulk RCMC. $K_{\text {mref }}, J_{m r e f}$, and $L_{m r e f}$ are the frequency quadratic, cubic, and quartic modulation of the reference target in the range-Doppler domain respectively, which is defined in Equation (A11). After this operation, the RCM values of all targets have been corrected, and the residual phase can be compensated by multiplying the echo with:

$$
\begin{aligned}
H_{\text {residual }}\left(f_{t}\right)=\exp \{ & j \pi\left(K_{\text {mref }}+K_{s 1} \cdot \tau^{\prime}+K_{s 2} \cdot \tau^{\prime 2}\right)\left(\alpha \cdot \tau^{\prime}+\beta \cdot{\tau^{\prime 2}}^{2}\right\} \\
\cdot & \exp \left\{-j \frac{2 \pi}{3}\left(J_{\text {mref }}+J_{s 1} \cdot \tau^{\prime}\right)\left(\alpha \cdot \tau^{\prime}+\beta \cdot \tau^{\prime 2}\right)^{3}\right\} \\
\cdot & \exp \left\{j \frac{\pi}{2} L_{\text {mref }}\left(\alpha \cdot \tau^{\prime}+\beta \cdot \tau^{\prime 2}\right)^{4}\right\} \\
\cdot & \exp \left\{j \pi\left[Q_{2} \cdot \tau^{\prime 2}+\frac{2}{3} Q_{3} \cdot \tau^{\prime 3}+\frac{1}{2} Q_{4} \cdot \tau^{\prime \prime}\right]\right\}
\end{aligned}
$$

where $\tau^{\prime}=\tau-2 r_{0, r e f} / c$ and $K_{s 1}$ and $K_{s 2}$ are the linear and quadratic coefficient of frequency modulation, respectively, which are defined in Equation (A11). $J_{s 1}$ is the linear coefficient of cubic modulation, and $\alpha$ and $\beta$ are the linear and quadratic scaling parameter, respectively, which can be obtained by traditional NCS method. The specific expression is also in Equation (A11). Here, the time-scaling operations in Equation (A1) have changed the spatial variance in the azimuth direction. Therefore, to facilitate azimuth processing, it was necessary to recover the spatial variance at each range bin by applying the filter

$$
H_{-} A S 1(t)=\exp \left\{j \frac{4 \pi}{\lambda} r_{p t}(t)\right\} \text {. }
$$

The resulting return signal has the form provided in Equation (17). 
Lastly, the specific expressions of the parameters given in Equations (A2)-(A5) are listed here. First, the 2D frequency domain phase is expanded in the following series form:

$$
\Phi\left(f_{t}, f_{\tau}\right) \approx 2 \pi \phi_{0}\left(f_{t}\right)+2 \pi \sum_{n=1}^{9} \phi_{n}\left(f_{t}\right) \cdot f_{\tau}^{n},
$$

where the coefficients $\phi_{n}\left(f_{t}\right)(n=0-9)$ are defined as follows:

$$
\left\{\begin{array}{rl}
\phi_{0}\left(f_{t}\right) & \approx \phi_{0, \text { ref }}\left(f_{t}\right)+M_{1, r}\left(f_{t}\right) \cdot \Delta R+M_{2, r}\left(f_{t}\right) \cdot \Delta R^{2}+M_{1, a}\left(f_{t} ; \Delta R\right) \cdot \Delta t \\
& +M_{2, a}\left(f_{t} ; \Delta R\right) \cdot \Delta t^{2}+M_{3, a}\left(f_{t} ; \Delta R\right) \cdot \Delta t^{3} \\
\phi_{1}\left(f_{t}\right) \approx \phi_{1, r e f}\left(f_{t}\right)+L_{1, r}\left(f_{t}\right) \cdot \Delta R+L_{2, r}\left(f_{t}\right) \cdot \Delta R^{2}+L_{1, a}\left(f_{t} ; \Delta R\right) \cdot \Delta t & \\
& +L_{2, a}\left(f_{t} ; \Delta R\right) \cdot \Delta t^{2} \\
\phi_{2}\left(f_{t}\right) \approx & \phi_{2, r e f}\left(f_{t}\right)+J_{1, r}\left(f_{t}\right) \cdot \Delta R+J_{1, a}\left(f_{t} ; \Delta R\right) \cdot \Delta t+J_{2, a}\left(f_{t} ; \Delta R\right) \cdot \Delta t^{2} \\
\phi_{3}\left(f_{t}\right) \approx \phi_{3, \text { ref }}\left(f_{t}\right)+K_{1, r}\left(f_{t}\right) \cdot \Delta R+K_{1, a}\left(f_{t} ; \Delta R\right) \cdot \Delta t \\
\phi_{k}\left(f_{t}\right) \approx \phi_{k, \text { ref }}\left(f_{t}\right), 4 \leq k \leq 9
\end{array} .\right.
$$

This is the spatial mode of the 2D spectrum, and the specific expressions employed herein are listed in Table A1.

\begin{tabular}{|c|c|}
\hline$\phi_{0}\left(f_{t}\right)$ & $\phi_{1}\left(f_{t}\right)$ \\
\hline $\begin{array}{l}\phi_{0, r e f}\left(f_{t}\right)=-\frac{2 r_{0, \text { ref }}}{\lambda}+\sum_{m=2}^{10} \frac{A_{m-1, \text { ref }}}{m}\left(-\frac{\lambda f_{t}}{2}\right)^{m} \\
M_{1, r}\left(f_{t}\right)=-\frac{2}{\lambda}+\sum_{m=2}^{10} \frac{l_{m-1,1, r}}{m}\left(-\frac{\lambda f_{t}}{2}\right)^{m} \\
M_{2, r}\left(f_{t}\right)=\frac{2}{\lambda} \sum_{m=2}^{10} \frac{l_{m-1,2, r}}{m}\left(-\frac{\lambda f_{t}}{2}\right)^{m} \\
M_{1, a}\left(f_{t} ; \Delta R\right)=\frac{2}{\lambda} \sum_{m=2}^{10} \frac{l_{m-1,1, a}}{m}\left(-\frac{\lambda f_{t}}{2}\right)^{m} \\
M_{2, a}\left(f_{t} ; \Delta R\right)=\frac{2}{\lambda} \sum_{m=2}^{10} \frac{l_{m-1,2, a}}{m}\left(-\frac{\lambda f_{t}}{2}\right)^{m} \\
M_{3, a}\left(f_{t} ; \Delta R\right)=\frac{2}{\lambda} \sum_{m=2}^{10} \frac{l_{m-1,3, a}}{m}\left(-\frac{\lambda f_{t}}{2}\right)^{m}\end{array}$ & $\begin{array}{l}\phi_{1, r e f}\left(f_{t}\right)=-\frac{2 r_{0, r e f}}{c}-\frac{2}{\lambda} \sum_{m=2}^{10} \frac{A_{m-1, \text { ref }}}{m}(m-1)\left(-\frac{\lambda f_{t}}{2}\right)^{m} \\
L_{1, r}\left(f_{t}\right)=-\frac{2}{c}-\frac{2}{\lambda} \sum_{m=2}^{10} \frac{l_{m-1,1, r}}{m}(m-1)\left(-\frac{\lambda f_{t}}{2}\right)^{m} \\
L_{2, r}\left(f_{t}\right)=-\frac{2}{c} \sum_{m=2}^{10} \frac{l_{m-1,2, r}}{m}(m-1)\left(-\frac{\lambda f_{t}}{2}\right)^{m} \\
L_{1, a}\left(f_{t}\right)=-\frac{2}{c} \sum_{m=2}^{10} \frac{l_{m-1,1, a}}{m}(m-1)\left(-\frac{\lambda f_{t}}{2}\right)^{m} \\
L_{2, a}\left(f_{t}\right)=-\frac{2}{c} \sum_{m=2}^{10} \frac{l_{m-1,2, a}}{m}(m-1)\left(-\frac{\lambda f_{t}}{2}\right)^{m}\end{array}$ \\
\hline$\phi_{2}\left(f_{t}\right)$ & $\phi_{3}\left(f_{t}\right)$ \\
\hline $\begin{array}{l}\phi_{2, r e f}\left(f_{t}\right)=-\frac{1}{2 K_{r}}+\frac{2}{c f_{0}} \sum_{m=2}^{10} \frac{A_{m-1, r e f}}{m} C_{m}^{2}\left(-\frac{\lambda f_{t}}{2}\right)^{m} \\
J_{1, r}\left(f_{t}\right)=\frac{2}{c f_{0}} \sum_{m=2}^{10} \frac{l_{m-1,1, r}}{m} C_{m}^{2}\left(-\frac{\lambda f_{t}}{2}\right)^{m} \\
J_{1, a}\left(f_{t} ; \Delta R\right)=\frac{2}{c f_{0}} \sum_{m=2}^{10} \frac{l_{m-1,1, a}}{m} C_{m}^{2}\left(-\frac{\lambda f_{t}}{2}\right)^{m} \\
J_{2, a}\left(f_{t} ; \Delta R\right)=\frac{2}{c f_{0}} \sum_{m=2}^{10} \frac{l_{m-1,2, a}}{m} C_{m}^{2}\left(-\frac{\lambda f_{t}}{2}\right)^{m}\end{array}$ & $\left\{\begin{array}{l}\phi_{3, \text { ref }}\left(f_{t}\right)=-\frac{2}{c f_{0}^{2}} \sum_{m=2}^{10} \frac{A_{m-1, \text { ref }}}{m} C_{m+1}^{3}\left(-\frac{\lambda f_{t}}{2}\right)^{m} \\
K_{1, r}\left(f_{t}\right)=-\frac{2}{c f_{0}^{2}} \sum_{m=2}^{10} \frac{l_{m-1,1, r}}{m} C_{m+1}^{3}\left(-\frac{\lambda f_{t}}{2}\right)^{m} \\
K_{1, a}\left(f_{t}\right)=-\frac{2}{c f_{0}^{2}} \sum_{m=2}^{10} \frac{l_{m-1,1, a}}{m} C_{m+1}^{3}\left(-\frac{\lambda f_{t}}{2}\right)^{m}\end{array}\right.$ \\
\hline \multicolumn{2}{|l|}{$\phi_{k}\left(f_{t}\right), 4 \leq k \leq 9$} \\
\hline$\phi_{k, \text { ref }}\left(f_{t}\right)=(-1)^{k} \frac{2}{c} \sum_{m=2}^{10} \frac{A_{m-1, \text { ref }}}{m} C_{m+k-2}^{k}\left(-\frac{\lambda f_{t}}{2}\right)^{m}$ & \\
\hline
\end{tabular}

Table A1. Spatially variant coefficients in the 2D frequency domain.

In these expressions, $C_{m}^{k}=m ! /[k !(m-k) !]$ is the combinational symbol, and $A_{m}(m=1-9)$ are the coefficients obtained by series reversion [23], which are given as follows: 


$$
\left\{\begin{array}{l}
A_{1}=1 / r_{2} \\
A_{2}=-r_{3} / 2 r_{2}^{3} \\
A_{3}=\left(3 r_{3}^{2}-r_{2} r_{4}\right) / 6 r_{2}^{5} \\
A_{4}=-\left(r_{2}^{2} r_{5}+15 r_{3}^{3}-10 r_{2} r_{3} r_{4}\right) / 24 r_{2}^{7} \\
A_{5}=\left(10 r_{2}^{2} r_{4}^{2}-r_{2}^{3} r_{6}+105 r_{2} r_{3}^{2} r_{4}-105 r_{2} r_{3}^{2} r_{4}+15 r_{2}^{2} r_{3} r_{5}\right) / 120 r_{2}^{9} \\
A_{6}=-\left(r_{2}^{4} r_{7}-21 r_{2}^{3} r_{3} r_{6}-35 r_{2}^{3} r_{4} r_{5}+210 r_{2}^{2} r_{3}^{2} r_{5}+280 r_{2}^{2} r_{3} r_{4}^{2}-1260 r_{2} r_{3}^{3} r_{4}+945 r_{3}^{5}\right) / 720 r_{2}^{11} \\
\cdots
\end{array}\right.
$$

In addition, $\left\{l_{m, 1, r}, l_{m, 2, r}, l_{m, 1, a}, l_{m, 2, a}, l_{m, 3, a}\right\}$ are the spatial coefficients, which can be resolved by numerical analysis according to the function

$$
A_{m}=A_{m, r e f}+l_{m, 1, r} \cdot \Delta R+l_{m, 2, r} \cdot \Delta R^{2}+l_{m, 1, a} \cdot \Delta t+l_{m, 2, a} \cdot \Delta t^{2}+l_{m, 3, a} \cdot \Delta t^{3} .
$$

Expressions for the parameters related to CS in Equations (A2)-(A5) can be determined according to a previously proposed method [21], and are given as follows:

$$
\left\{\begin{array}{l}
K_{\text {mref }}=1 /\left(2 \phi_{2, \text { ref }}\right) \\
K_{s 1}=2 K_{m r e f}^{2} J_{1, r}\left(f_{t}\right) / L_{1, r}\left(f_{r e f}\right) \\
K_{s 2}=\left[4 K_{m r e f}^{3} J_{1, r}^{2}\left(f_{t}\right)-2 K_{m r e f}^{2} J_{2, r}\left(f_{t}\right)\right] / L_{1, r}^{2}\left(f_{r e f}\right)+2 K_{m r e f}^{2} J_{1, r}\left(f_{t}\right) L_{2, r}\left(f_{r e f}\right) / L_{1, r}^{3}\left(f_{r e f}\right) \\
J_{m r e f}=\left[2 \beta K_{m r e f}+(2 \alpha+1) K_{s 1}\right] / 2 \alpha(\alpha+1) \\
J_{s 1}=\left[6 J_{1, r}\left(f_{t}\right) \cdot J_{m r e f} \cdot K_{m r e f}-3 K_{1, r}\left(f_{t}\right) \cdot K_{m r e f}^{3}\right] / L_{1, r}\left(f_{r e f}\right) \\
L_{m r e f}=\left[(2 \alpha+1) J_{s 1}+2 J_{m r e f} \beta-K_{s 2}\right] / 3 \alpha(\alpha+1) \\
\alpha=L_{1, r}\left(f_{t}\right) / L_{1, r}\left(f_{r e f}\right)-1 \\
\beta=\left[L_{1, r}\left(f_{t}\right) L_{2, r}\left(f_{r e f}\right)-L_{2, r}\left(f_{t}\right) L_{1, r}\left(f_{r e f}\right)\right] / L_{1, r}^{3}\left(f_{r e f}\right) \\
Y=J_{m r e f} / K_{m r e f}^{3}-3 \phi_{3, r e f} \\
Z=\left(2 J_{m r e f}^{2}-L_{m r e f} K_{m r e f}\right) / K_{m r e f}^{5} \\
Q_{2}=\alpha K_{m r e f} \\
Q_{3}=\left(0.5 \alpha K_{s}+\beta K_{m r e f}\right) /(1+\alpha) \\
Q_{4}=\alpha L_{m r e f}-J_{s 1} / 3
\end{array}\right.
$$

\section{Appendix B.}

In Equations (19) and (20), the parameters $Y_{3}, Y_{4}, p_{3}, p_{4}$, and $p_{5}$ can be derived according to a previous study [16] as follows:

$$
\left.\begin{array}{c}
Y_{3}=\frac{1}{4 k_{2,1, a} \cdot\left(\left.r_{2}\right|_{t_{c}=0}\right)^{3}}\left[-k_{2,1, a}^{2}-\left.r_{3}\right|_{t_{c}=0} \cdot k_{2,1, a}-\left.\left(2 k_{2,2, a}+3 k_{3,1, a}\right) \cdot r_{2}\right|_{t_{c}=0}\right] \\
Y_{4}=-\frac{1}{60 k_{2,1, a}^{2}\left(\left.r_{2}\right|_{t_{c}=0}\right)^{5}}\left[\begin{array}{l}
12 k_{2,1, a}^{2} \cdot\left(\left.r_{3}\right|_{t_{c}=0}\right)^{2}-15 k_{2,2, a}^{2} \cdot\left(\left.r_{3}\right|_{t_{c}=0}\right)^{2}-\left.21 k_{2,1, a}^{3} \cdot r_{3}\right|_{t_{c}=0} \\
+9 k_{2,1, a}^{4}-\left.24 k_{2,1, a}^{2} \cdot k_{2,2, a} \cdot r_{2}\right|_{t_{c}=0}+\left.11 k_{2,1, a}^{2} \cdot k_{3,1, a} \cdot r_{2}\right|_{t_{c}=0} \\
-6 k_{2,1, a} \cdot k_{3,2, a} \cdot\left(\left.r_{2}\right|_{t_{c}=0}\right)^{2}-6 k_{2,2, a} \cdot k_{3,1, a} \cdot\left(\left.r_{2}\right|_{t_{c}=0}\right)^{2} \\
-\left.\left.2 k_{2,1, a}^{2} \cdot r_{2}\right|_{t_{c}=0} \cdot r_{4}\right|_{t_{c}=0}+\left.30 k_{2,1, a} \cdot k_{2,2, a} \cdot r_{2}\right|_{t_{c}=0}=0 \\
-\left.\left.12 k_{c, 1, a} \cdot k_{3,1, a} \cdot r_{2}\right|_{t_{c}=0} \cdot r_{3}\right|_{t_{c}=0}
\end{array}\right] \\
p_{3}=-\left.k_{2,1, a}\right|_{\Delta R} \\
p_{4}=-k_{3,1, a}-\left.3 Y_{3} k_{2,1, a} \cdot r_{2}\right|_{t_{c}=0} ^{2}
\end{array}\right]
$$




$$
p_{5}=-k_{4,1, a}-\left.6 Y_{3} k_{3,1, a} \cdot r_{2}\right|_{t_{c}=0} ^{2}-\left.24 Y_{4} k_{2,1, a} \cdot r_{2}\right|_{t_{c}=0} ^{3}-\left.15 Y_{3}^{2} k_{2,1, a} \cdot r_{2}\right|_{t_{c}=0} ^{4}-\left.\left.12 Y_{3} k_{2,1, a} \cdot r_{2}\right|_{t_{c}=0} \cdot r_{3}\right|_{t_{c}=0}
$$

\section{Appendix C.}

The parameters $P_{m}(m=3-10)$ have the form

$$
P_{m}=(-1)^{m} A_{m-1} / m(m \geq 2),
$$

where $A_{m}$ was defined in Equation (A9).

\section{References}

1. Tomiyasu, K. Synthetic aperture radar in geosynchronous orbit. In Proceedings of the 1978 Antennas and Propagation Society International Symposium, Washington, DC, USA, 9-13 March 1978; pp. 42-45. [CrossRef]

2. Tomiyasu, K.; Pacelli, J.L. Synthetic Aperture Radar Imaging from an Inclined Geosynchronous Orbit. IEEE Trans. Geosci. Remote Sens. 1983, GE-21, 324-329. [CrossRef]

3. Global Earthquake Satellite System: A 20-Year Plan to Enable Earthquake Prediction, JPL Document, NASA and JPL, Tech. Rep. JPL 400-1069. 2003. Available online: https://solidearth.jpl.nasa.gov/GESS/3123_ GESS_Rep_2003.pdf (accessed on 18 April 2003).

4. Madsen, S.N.; Edelstein, W.; DiDomenico, L.D.; LaBrecque, J. A geosynchronous synthetic aperture radar; for tectonic mapping, disaster management and measurements of vegetation and soil moisture. In Proceedings of the IGARSS 2001, Sydney, Australia, 9-13 July 2001; Volume 1, pp. 447-449. [CrossRef]

5. Li, D.; Wu, M.; Sun, Z.; He, F.; Dong, Z. Modeling and Processing of Two-Dimensional Spatial-Variant Geosynchronous SAR Data. IEEE J. Sel. Top. Appl. Earth Obs. Remote Sens. 2015, 8, 3999-4009. [CrossRef]

6. Li, Z.; Li, C.; Yu, Z.; Zhou, J.; Chen, J. Back projection algorithm for high resolution GEO-SAR image formation. In Proceedings of the IEEE International Geoscience and Remote Sensing Symposium, Vancouver, BC, Canada, 24-29 July 2011; pp. 336-339. [CrossRef]

7. Ulander, L.M.H.; Hellsten, H.; Stenstrom, G. Synthetic-aperture radar processing using fast factorized back-projection. IEEE Trans. Aerosp. Electron. Syst. 2003, 39, 760-776. [CrossRef]

8. Cafforio, C.; Prati, C.; Rocca, F. SAR data focusing using seismic migration techniques. IEEE Trans. Aerosp. Electron. Syst. 1991, 27, 194-207. [CrossRef]

9. Raney, R.K.; Runge, H.; Bamler, R.; Cumming, I.G.; Wong, F.H. Precision SAR processing using chirp scaling. IEEE Trans. Geosci. Remote Sens. 1994, 32, 786-799. [CrossRef]

10. Franceschetti, G.; Lanari, R.; Marzouk, E.S. A new two-dimensional squint mode SAR processor. IEEE Trans. Aerosp. Electron. Syst. 1996, 32, 854-863. [CrossRef]

11. Bao, M.; Xing, M.D.; Li, Y.C. Chirp scaling algorithm for GEO SAR based on fourth-order range equation. Electron. Lett. 2012, 48, 41-42. [CrossRef]

12. Hu, C.; Long, T.; Tian, Y. An Improved Nonlinear Chirp Scaling Algorithm Based on Curved Trajectory in Geosynchronous SAR. Prog. Electromagn. Res. 2013, 135, 481-513. [CrossRef]

13. Zeng, T.; Yang, W.; Ding, Z.; Liu, D.; Long, T. A Refined Two-Dimensional Nonlinear Chirp Scaling Algorithm for Geosynchronous Earth Orbit SAR. Prog. Electromagn. Res. 2013, 143, 19-46. [CrossRef]

14. Sun, G.; Xing, M.; Wang, Y.; Yang, J.; Bao, Z. A 2-D Space-Variant Chirp Scaling Algorithm Based on the RCM Equalization and Subband Synthesis to Process Geosynchronous SAR Data. IEEE Trans. Geosci. Remote Sens. 2014, 52, 4868-4880. [CrossRef]

15. Chen, J.; Sun, G.; Wang, Y.; Xing, M.; Li, Z.; Zhang, Q.; Liu, L.; Dai, C. A TSVD-NCS Algorithm in Range-Doppler Domain for Geosynchronous Synthetic Aperture Radar. IEEE Geosci. Remote Sens. Lett. 2016, 13, 13-1635. [CrossRef]

16. Yu, Z.; Lin, P.; Xiao, P.; Kang, L.; Li, C. Correcting spatial variance of RCM for geo sar imaging based on time-frequency scaling. Sensors 2016, 16, 1091. [CrossRef]

17. Ding, Z.; Shu, B.; Yin, W.; Zeng, T.; Long, T. A Modified Frequency Domain Algorithm Based on Optimal Azimuth Quadratic Factor Compensation for Geosynchronous SAR Imaging. IEEE J. Sel. Top. Appl. Earth Obs. Remote Sens. 2016, 9, 1119-1131. [CrossRef] 
18. Zhang, T.; Ding, Z.; Tian, W.; Zeng, T.; Yin, W. A 2-D Nonlinear Chirp Scaling Algorithm for High Squint GEO SAR Imaging Based on Optimal Azimuth Polynomial Compensation. IEEE J. Sel. Top. Appl. Earth Obs. Remote Sens. 2017, 10, 5724-5735. [CrossRef]

19. Motohka, T.; Kankaku, Y.; Suzuki, S.; Shimada, M. Status of the advanced land observing satellite-2 (ALOS-2) and its follow-on L-band SAR mission. In Proceedings of the IEEE International Geoscience and Remote Sensing Symposium (IGARSS), Fort Worth, TX, USA, 23-28 July 2017; pp. 2427-2429. [CrossRef]

20. Cumming, I.G.; Wong, F.H. Digital Signal Processing of Synthetic Aperture Radar Data; Artech House: Norwood, MA, USA, 2004.

21. Geng, J.; Yu, Z.; Lin, P.; Xiao, P.; Li, C. Fourth-order chirp scaling imaging algorithm for Geosynchronous Synthetic Aperture Radar. In Proceedings of the EUSAR 2018, 12th European Conference on Synthetic Aperture Radar, Aachen, Germany, 4-7 June 2018; pp. 1-5.

22. Davidson, G.W.; Cumming, I.G.; Ito, M.R. A chirp scaling approach for processing squint mode SAR data. IEEE Trans. Aerosp. Electron. Syst. 1996, 32, 121-133. [CrossRef]

23. Neo, Y.L.; Wong, F.; Cumming, I.G. A Two-Dimensional Spectrum for Bistatic SAR Processing Using Series Reversion. IEEE Geosci. Remote Sens. Lett. 2007, 4, 93-96. [CrossRef]

(C) 2018 by the authors. Licensee MDPI, Basel, Switzerland. This article is an open access article distributed under the terms and conditions of the Creative Commons Attribution (CC BY) license (http://creativecommons.org/licenses/by/4.0/). 